T. Higa

Nagoya Math. J.

Vol. 72 (1978), 27-63

\title{
LES EQUATIONS DIFFERENTIELLES INVARIANTES PAR LES PSEUDO-GROUPES
}

\author{
TATSUO HIGA
}

\section{Introduction}

S. Lie a étudié méthodiquement, pour la première fois, des propriétés des équations différentielles invariantes par les groupes continus de transformations locales (pseudo-groupes continus). Après avoir étudié la structure des pseudo-groupes, Lie a esquissé la méthode générale d'intégration des équations différentielles, [9]. Un de ses idées fondamentales est celle de réduire les équations différentielles aux autres équations faciles à étudier : par exemple, les systèmes de Pfaff complètement intégrables.

La détermination d'un pseudo-groupe laissant invariant l'équation différentielle donnée est, en général, très difficile et, en outre, il y a beaucoup d'équations différentielles (non-linéaires) qui n'admettent que l'identité d'un espace.

Cependant, en Géométrie différentielle ou Mécanique, on peut rencontrer souvent des équations différentielles invariantes par les pseudogroupes continus dont la structure est bien connue ou facile à étudier (cf. [1], [3]). Par exemple: L'équation différentielle déterminée par le problème d'existence d'immersions isométriques locales d'une variété riemannienne dans une autre; elle est invariante par le pseudo-groupe fini des isométries locales des deux variétés riemanniennes (cf. [2], [4]). L'équations différentielles parues dans la Géométrie des $G$-structures, [10], [11]; la plupart de ces équations peuvent être envisagées comme des équations invariantes par certains pseudo-groupes.

Le problème que nous traitons dans ce Mémoire est le suivant. Etant donnés un pseudo-groupe continu fini ou infini $\mathscr{L}$ et une équation différentielle $R$ invariante par $\mathscr{L}$, nous voulons étudier d'abord les conditions moyennant lesquelles l'équation $R$ puisse être réduite à certains

Received June 30, 1976. 
systèmes de Pfaff complètement intégrables ; ensuite le problème d'existence de solutions locales de $R$. Les conditions obtenues doivent être géométriques ou algébriques. Nous voulons en fait unifier les divers théorèmes parus dans la Géométrie différentielle.

Dans le chapitre I, nous posons la définition des équations différentielles aux dérivées partielles, et ses solutions locales. Pour simplifier les idées, nous étudierons principalement les équations différentielles du premier ordre.

Dans le chapitre II, nous traitons la structure des pseudo-groupes laissant invariant les équations. Au lieu des pseudo-groupes, nous considérerons les faisceaux en algèbres de Lie (transitifs et $\pi$-projetables) dont la théorie est développée dans [11] et les G-structures associées aux faisceaux. Il existe toujours une équation différentielle invariante par $\mathscr{L}$ (Théorèmes 2.1,2.2).

Pour un faisceau en algèbres de Lie transitif et $\pi$-projetable $\mathscr{L}$, nous pouvons définir une certaine constante structurale $C_{\sigma}$ et un sousespace vectoriel $\phi_{\sigma}(\mathrm{g})$ de Hom $\left(\boldsymbol{R}^{n}, \boldsymbol{R}^{m}\right), \mathfrak{g}$ étant l'algèbre d'isotropie linéaire de $\mathscr{L}$ (voir $\S 9$ et $\S 11$ ).

Dans le chapitre III, les théorèmes suivants sont démontrés. Soit $\mathscr{L}$ un faisceau en algèbres de Lie transitif et $\pi$-projetable et soit $R$ une équation différentielle du premier ordre invariante par $\mathscr{L}$.

1) Si $\mathscr{L}$ est plat, $R$ peut être réduite à certains systèmes de Pfaff complètement intégrables (Théorème 3.1).

2) Si $\mathscr{L}$ vérifie les conditions i) $C_{\sigma}=0$, ii) $\phi_{\sigma}(\mathfrak{g})=0, R$ peut être réduite à certains systèmes de Pfaff complètement intégrables (Théorèmes $3.2,3.3)$.

3) Supposons que $\mathscr{L}$ et $R$ soient analytiques réels. Si $\mathscr{L}$ vérifie les conditions i) $C_{\sigma}=0$, ii) $\phi_{\sigma}(\mathfrak{g})$ est involutif, il existe, pour tout $y \in M$ (l'espace de variables indépendantes et dépendantes), une solution locale de $R$ passant par le point $y$ (Théorèmes $3.4,3.5$ ).

Dans le paragraphe 13, nous appliquons la théorie générale à la Géométrie différentielle locale. Par exemple, nous pouvons retrouver le théorème de Singer-Sternberg en utilisant le théorème 3) ci-dessus (voir [11] Chap. III) :

THÉORغ̀me 3.6. Soient $B_{1}, B_{2}$ des G-structures sur $N_{1}, N_{2}$ respectivement. Si les conditions suivantes sont vérifiées; 
i) les fonctions structurales $C_{1}$ et $C_{2}$ sont constantes et $C_{1}=C_{2}$,

ii) l'algèbre de Lie de $G$ est involutive, alors $B_{1}$ et $B_{2}$ sont localement isomorphes.

Dans toute la suite, $\boldsymbol{R}^{n}$ désigne l'espace vectoriel réel canonique à $n$ dimensions. Le mot «différentiable» signifie «différentiable de classe $C^{\infty}$ ». Mais, à partir du paragraphe 12, nous supposons que tous les objets soient analytiques réels. Soit $M$ une variété, indiquons par $T_{x}(M)$ l'espace vectoriel tangent à $M$ en $x \in M$. Soit $f$ une application différentiable d'une variété $U(f)$ dans une autre $V(f), f_{*}$ désigne l'application tangente de $f$ et $f^{*}$ l'application cotangente.

Nous voulons exprimer ici notre profonde reconnaissance au Professeur A. Morimoto qui nous a donné les conseils bienveillants.

\section{Equations différentielles aux dérivées partielles}

1 Nous allons commencer par poser la définition des équations différentielles sur une variété différentiable. Dans toute la suite de ce chapitre, $M$ et $N$ désigneront des variétés différentiables et $\pi: M \rightarrow N$ une fibration (submersion surjective).

Nous indiquons par $J^{k}(M, \pi)(k=1,2, \cdots)$ la variété des $k$-jets de sections locales de $\pi$. Soit $s$ une section locale de $\pi$ et désignons par $j_{x}^{k}(s)$ le $k$-jet de $s$ en $x \in U(s)$ (le domaine de $s$ ). Les applications source $\alpha^{k}: J^{k}(M, \pi) \rightarrow N$ et but $\beta^{k}: J^{k}(M, \pi) \rightarrow M$ sont définies par les formules : $\alpha^{k}\left(j_{x}^{k}(s)\right)=x, \beta^{k}\left(j_{x}^{k}(s)\right)=s(x)$, et il se trouve que $\alpha^{k}$ et $\beta^{k}$ sont des submersions surjectives. Donc, pour une section $s,\left(j^{k} s\right)(x)=j_{x}^{k}(s)$ donne une section locale de $\alpha^{k}$. Lorsque $M$ est un fibré vectoriel sur $N, J^{k}(M, \pi)$ admet une structure canonique de fibré vectoriel sur $N$.

Si $k=1$, nous pouvons envisager $J^{1}(M, \pi)$ comme l'espace $E$ de toutes les applications linéaires injectives de la forme suivante;

$$
z: T_{x}(N) \rightarrow T_{y}(M), \quad \pi(y)=x, \quad \pi_{*} \circ z=\mathrm{id} .
$$

Plus exactement, comme l'élément $j_{x}^{1}(s)$ est entièrement déterminé par $x, s(x)$ et les valeurs en $x$ des dérivées du premier ordre de $s$, on peut définir l'application linéaire $z$ de $T_{x}(N)$ sur $T_{s(x)}(M)$ par $z=\left(s_{*}\right)_{x}$. Donc nous envisagerons $J^{1}(M, \pi)$ soit comme l'espace des 1 -jets soit comme l'espace $E$ ci-dessus.

Supposons maintenant que $M$ soit le produit de $N$ et une autre 
variété différentiable $Q$, i.e., $M=N \times Q$ et que $\pi$ soit la projection canonique $N \times Q \rightarrow N$. Lorsque ceci est le cas, $J^{k}(M, \pi)$ s'identifie avec la variété des $k$-jets des applications différentiables locales de $N$ dans $Q$, i.e., $J^{k}(N, Q)$ et $J^{1}(M, \pi)$ s'identifie, cette fois-ci, avec l'espace de toutes les applications linéaires de $T_{x}(N)$ dans $T_{q}(Q)$ (pour tous $x \in N$, $q \in Q)$.

Soit $M^{\prime}$ une variété différentiable et soit $\pi^{\prime}: M^{\prime} \rightarrow N$ une fibration. Supposons que $M^{\prime}$ soit un sous-fibré de $M$ par rapport à $\pi$, alors $J^{k}\left(M^{\prime}, \pi^{\prime}\right)$ est considéré comme un sous-fibré de $J^{k}(M, \pi)$ par rapport à la fibration $\alpha^{k}: J^{k}(M, \pi) \rightarrow N$.

DÉFinition 1.1 (cf. [5], [6]). Nous disons qu'une sous-variété $R_{k}$ de $J^{k}(M, \pi)$ est une équation différentielle aux dérivées partielles d'ordre $k$ sur $(M, \pi, N)$ si et seulement si $R_{k}$, muni de cette structure de variété, est un sous-fibré de $J^{k}(M, \pi)$ par rapport à la fibration $\alpha^{k}$.

Remarque. 1) Si $R_{k}$ est un sous-fibré différentiable de $J^{k}(M, \pi)$ par rapport à la fibration $\beta^{k}, R_{k}$ est considéré comme une équation différentielle d'ordre $k$. Cela signifie que $R_{k}$ ne donne aucune relation d'ordre zéro entre les fonctions inconnues.

2) Lorsque $M$ est un fibré vectoriel sur $N$ et $\dot{R}_{k}$ est un sous-fibré vectoriel de $J^{k}(M, \pi), R_{k}$ est appelé une équation différentielle linéaire d'ordre $k$ (cf. [5]).

3) L'équation différentielle $R_{k}$ dans la définition est localement donnée par l'ensemble de tous les points qui annulent un nombre fini de fonctions différentiables locales. $\mathrm{Si}$, en outre, $R_{k}$ satisfait à une condition convenable, il existe un fibré différentiable sur $N, \pi^{\prime}: M^{\prime} \rightarrow N$, un morphisme de fibrés $\psi: J^{k}(M, \pi) \rightarrow M^{\prime}$ et une section différentiable $s^{\prime}$ de $\pi^{\prime}$ tels que

i) $s^{\prime}(N) \subset \psi\left(J^{k}(M, \pi)\right)$,

ii) $R_{k}=\left\{z \in J^{k}(M, \pi) \mid \psi(z)=s^{\prime}\left(\alpha^{k}(z)\right)\right\}$

soient vérifiés (voir [6]).

Proposition 1.1. Soit $M^{\prime}$ un sous-fibré de $M$ par rapport à $\pi$ et soit $R_{k}^{\prime}$ une équation différentielle d'ordre $k$ sur $\left(M^{\prime},\left.\pi\right|_{M^{\prime}}, N\right)$. Alors $R_{k}^{\prime}$ est considéré comme une équation différentielle d'ordre $k$ sur $(M, \pi, N)$.

DÉfinItion 1.2. Soit $R_{k}$ une équation différentielle d'ordre $k$ sur 
$(M, \pi, N)$. Une section différentiable locale $s$ de $\pi$ définie sur un ouvert $V$ de $N$ est dit une solution locale de $R_{k}$ si et seulement si $\left(j^{k} s\right)(V) \subset R_{k}$ est vérifié.

2 Donnons quelques exemples. Soit $Q$ une variété différentiable.

EXEMPLe I. Supposons que $\operatorname{dim} N \leqq \operatorname{dim} Q$. Etant données une métrique riemannienne $g$ sur $N$ et $\tilde{g}$ sur $Q$, nous indiquons par $R(g, \tilde{g})$ l'ensemble de toutes les applications linéaires $z: T_{x}(N) \rightarrow T_{y}(Q)$ (pour tous $x \in N, y \in Q)$ telles que $\tilde{g}_{y}(z(X), z(Y))=g_{x}(X, Y)$ pour tous $X, Y \in T_{x}(N)$. On voit aisément que $R(g, \tilde{g})$ est une équation différentielle du premier ordre sur $(N \times Q, \pi, N)$ et qu'une application différentiable locale $f$ de $N$ dans $Q$ est une solution locale de $R(g, \tilde{g})$ si et seulement si $f$ vérifie $f^{*} \tilde{g}=g$, c'est-à-dire une immersion isométrique. Si $Q=E^{m}$, l'espace Euclidien de dimension $m$ avec la métrique canonique $d s$, l'existence de solutions locales de $R(g, d s)$ signifie que $N$ peut être localement plongé dans $\boldsymbol{E}^{m}$ et que, dans le cas particulier de $m=\operatorname{dim} N, N$ est un espace de Riemann localement euclidien.

La théorie de $G$-structures peut généraliser la situation ci-dessus aux autres structures géométriques. Soit $G$ un sous-groupe de Lie de $G L(n, \boldsymbol{R}), n$ étant la dimension de $N$; une $G$-structure $B$ sur $N$ est la donnée d'un sous-fibré principal, du groupe structural $G$, du fibré de repères de $N, \varpi: F(N) \rightarrow N$ (cf. [10]).

Exemple II. Supposons que $\operatorname{dim} N=\operatorname{dim} Q$. Etant données deux $G$-structures $B, \tilde{B}$ sur $N$ et $\operatorname{sur} Q$ respectivement, nous indiquons par $R(B, \tilde{B})$ l'ensemble de toutes les applications linéaires isomorphes $z$ de $T_{x}(N)$ sur $T_{y}(Q)$ (pour tous $x \in N, y \in Q$ ) vérifiant la condition $z\left(B_{x}\right)=\tilde{B}_{y}$, où $B_{x}$ (resp. $\tilde{B}_{y}$ ) désigne $B \cap \varpi^{-1}(x)$ (resp. $\tilde{B} \cap \widetilde{\varpi}^{-1}(y)$ ). Il se trouve que $R(B, \tilde{B})$ est une équation différentielle du premier ordre $\operatorname{sur}(N \times Q, \pi, N)$. L'existence de solutions locales signifie que $B$ et $\tilde{B}$ sont localement isomorphes. En particulier, si $B$ et $\tilde{B}$ sont des $G$-structures plates, il existe toujours, pour tous $x \in N, y \in Q$, une solution locale $s$ telle que $s(x)=y$ (cf. [10], [11]). Ce fait sera généralisé aux autres équations.

Exemple III. Etant données $r$ expressions de Pfaff linéairement indépendantes $\omega^{1}, \cdots, \omega^{r}$ sur $M(r<\operatorname{dim} M)$, nous indiquons par $R\left(\omega^{1}, \cdots\right.$, $\omega^{r}$ ) l'ensemble de tout point $z \in J^{1}(M, \pi)$ vérifiant $\omega^{i}(z(X))=0(i=1,2$, $\cdots, r)$ pour tout $X \in T_{\alpha(z)}(N) . \quad R\left(\omega^{1}, \cdots, \omega^{r}\right)$ ne devient pas toujours une 
équation différentielle.

Posons, pour tout $x \in N, M_{x}=\pi^{-1}(x)$ et désignons par $\iota_{x}$ l'inclusion de $M_{x}$ dans $M$. Considérons un système $\left(\omega^{1}, \cdots, \omega^{r}\right)$ satisfaisant à la condition;

(C) les formes induites $\iota_{x}^{*} \omega^{1}, \cdots, \iota_{x}^{*} \omega^{r}$ sur $M_{x}$ sont encore linéairement indépendantes pour tout $x \in N$.

Cela étant, nous pouvons prendre, pour tout $y \in M$, un système de coordonnées locales d'un voisinage ouvert $U$ de $y,\left(x^{1}, \cdots, x^{n}, y^{1}, \cdots, y^{m}\right)$ $(n=\operatorname{dim} N, n+m=\operatorname{dim} M)$, et celui de $V=\pi(U),\left(\bar{x}^{1}, \cdots, \bar{x}^{n}\right)$ tels que

i) $\bar{x}^{i} \circ \pi=x^{i} \quad(i=1,2, \cdots, n)$,

ii) $\widetilde{\varpi}^{i}=d y^{i}-\sum_{j=1}^{n} a_{j}^{i} d x^{j}-\sum_{k=r+1}^{m} b_{k}^{i} d y^{k}(i=1,2, \cdots, r)$ forment un système de Pfaff équivalent à $\left(\omega^{1}, \cdots, \omega^{r}\right)$, où $a_{j}^{i}, b_{k}^{i}$ sont des fonctions différentiables de $x^{i}$ et $y^{j}$.

Chaque élément $z \in \beta^{-1}(U) \subset J^{1}(M, \pi)$ est représenté par

$$
z\left(\frac{\partial}{\partial \bar{x}^{i}}\right)_{x}=\left(\frac{\partial}{\partial x^{i}}\right)_{y}+\sum_{j=1}^{m} p_{i}^{j}\left(\frac{\partial}{\partial y^{j}}\right)_{y} \quad(y=\beta(z), x=\alpha(z))
$$

et par suite, $\left(x^{1}, \cdots, x^{n}, y^{1}, \cdots, y^{m}, p_{i}^{j}\right)$ devient un système de coordonnées locales de $\beta^{-1}(U)$. Il est facile de démontrer à l'aide de ce système de coordonnées que $R\left(\omega^{1}, \cdots, \omega^{r}\right) \cap \beta^{-1}(U)$ coïncide avec l'ensemble de tous les points qui annulent les fonctions suivantes;

$$
F_{j}^{i}=p_{\jmath}^{i}-a_{j}^{i}-\sum_{k=r+1}^{m} b_{k}^{i} p_{j}^{k} \quad(i=1,2, \cdots, r ; j=1,2, \cdots, n) .
$$

Cela veut dire que $R\left(\omega^{1}, \cdots, \omega^{r}\right)$ est un sous-fibré de $J^{1}(M, \pi)$ par rapport à la fibration but $\beta: J^{1}(M, \pi) \rightarrow M$. Il est utile, pour la suite, d'exprimer ce résultat sous la forme suivante.

Proposition 1.2. Soient $\omega^{1}, \cdots, \omega^{r}$ des formes différentielles linéairement indépendantes sur $M$. Si $\left(\omega^{1}, \cdots, \omega^{r}\right)$ satisfait à la condition (C), alors $R\left(\omega^{1}, \cdots, \omega^{r}\right)$ est un sous-fibré de $J^{1}(M, \pi)$ par rapport à la fibration but $\beta$ et par suite, $R\left(\omega^{1}, \cdots, \omega^{r}\right)$ est une équation différentielle du premier ordre sur $(M, \pi, N)$.

Supposons que $R\left(\omega^{1}, \cdots, \omega^{r}\right)$ donne une équation différentielle sur $(M, \pi, N)$. Alors il est évident qu'une section locale $s$ de $\pi$ est une solution de $R\left(\omega^{1}, \cdots, \omega^{r}\right)$ si et seulement si $s$ donne une variété intégrale à $n$ dimensions du système de Pfaff $\omega^{1}=0, \cdots, \omega^{r}=0$. 
Cet exemple nous conduit à poser la définition suivante :

DÉFinition 1.3. Etant donnée, en général, une équation différentielle du premier ordre $R \operatorname{sur}(M, \pi, N)$, nous disons que $R$ est complètement réductible si et seulement si, pour tout $y \in M$, il existe un voisinage ouvert $U$ de $y$ dans $M$ et $r$ expressions de Pfaff linéairement indépendantes $\omega^{1}, \cdots, \omega^{r}$ sur $U(r \leqq \operatorname{dim} M-\operatorname{dim} N)$ telles que

i) $\omega^{1}, \cdots, \omega^{r}$ satisfont à la condition (C),

ii) $\omega^{1}, \cdots, \omega^{r}$ forment un système de Pfaff complètement intégrable,

iii) toute solution de $R\left(\omega^{1}, \cdots, \omega^{r}\right)$ est encore celle de $R$, i.e., $R\left(\omega^{1}, \cdots, \omega^{r}\right) \subset R$.

Remarquons qu'une telle équation possède toujours des solutions. On verra dans le chapitre III que cette notion pour les équations différentielles peut être caractérisée par des propriétés géométriques ou algébrique des pseudo-groupes laissant les invariant.

3 Soit $\rho^{k}: J^{k}(M, \pi) \rightarrow J^{k-1}(M, \pi)(k \geqq 2)$ la projection canonique et soit $R_{k}$ une équation différentielle d'ordre $k$ sur $(M, \pi, N)$. Supposons que le rang de $\left.\rho^{k}\right|_{R_{k}}$, la restriction de $\rho^{k}$ à $R_{k}$, soit constant sur $R_{k}$; on observera que l'image $\tilde{M}=\rho^{k}\left(R_{k}\right)$ devient un sous-fibré de $J^{k-1}(M, \pi)$ par rapport à la fibration source $\alpha^{k-1}$ et en outre que $R_{k}$ peut être envisagée comme une équation différentielle du premier ordre $\operatorname{sur}\left(M,\left.\alpha^{k-1}\right|_{\tilde{M}}, N\right)$. En raison de ce fait, dans toute la suite de ce Mémoire, nous ne considérerons que les équations différentielles du premier ordre sur $(M, \pi, N)$.

Soit $R$ une équation différentielle du premier ordre sur $(M, \pi, N)$. Définissons une forme différentielle extérieure de degré $1, \omega$, à valeurs dans le fibré vectoriel $\operatorname{Ker} \pi_{*}=\left\{v \in T(M) \mid \pi_{*} v=0\right\}$;

$$
\omega_{z}\left(X_{z}\right)=\beta_{*} X_{z}-z\left(\alpha_{*} X_{z}\right) \quad\left(z \in R, X_{z} \in T_{z}(R)\right),
$$

$\alpha$ étant l'application source et $\beta$ l'application but.

DÉfinition 1.4. Nous dirons que $\omega$ est la forme canonique sur $R$.

Soit $\iota: R G J^{1}(M, \pi)$ l'inclusion et soit $\varpi$ la forme canonique sur $J^{1}(M, \pi)$, il est immédiat de constater que $\iota^{*} \varpi=\omega$. Soient $U$ un ouvert de $M,\left(x^{1}, \cdots, x^{n}, y^{1}, \cdots, y^{m}\right)(n+m=\operatorname{dim} M)$ un système de coordonnées locales de $U$ et $\left(\bar{x}^{1}, \cdots, \bar{x}^{n}\right)(n=\operatorname{dim} N)$ celui de $V=\pi(U)$ tels que $\bar{x}^{i} \circ \pi$ $=x^{i}, i=1,2, \cdots, n$. Indiquons par $\left(x^{1}, \cdots, x^{n}, y^{1}, \cdots, y^{m}, p_{i}^{j}\right)$ le système 
de coordonnées locales de $\beta^{-1}(U)$ attaché à $\left(x^{1}, \cdots, x^{n}, y^{1}, \cdots, y^{m}\right)$ (cf. 1.1). Prenons une section locale $s$ de $\pi ; j^{1}(s)$ est représenté par

$$
\left(j^{1} s\right)(x)\left(\frac{\partial}{\partial \bar{x}^{i}}\right)_{x}=\left(\frac{\partial}{\partial x^{i}}\right)_{y}+\sum_{j=1}^{m} \frac{\partial s^{j}}{\partial \bar{x}^{i}}(x)\left(\frac{\partial}{\partial y^{j}}\right)_{y} \quad(y=s(x))
$$

en posant $s^{j}=y^{j} \circ s(j=1,2, \cdots, m)$. Examinons ensuite l'expression locale de la forme canonique $\varpi \operatorname{sur} J^{1}(M, \pi)$, on a

$$
\widetilde{\varpi}^{j}=d y^{j}-\sum_{i=1}^{n} p_{\imath}^{j} d x^{i} \quad(j=1,2, \cdots, m),
$$

en posant $\widetilde{\varpi}=\sum_{j=1}^{m} \widetilde{\varpi}^{j}\left(\frac{\partial}{\partial y^{j}}\right)$.

Considérons maintenant une section $\tau$ de $\left.\alpha\right|_{\beta-1(U)}: \beta^{-1}(U) \rightarrow V$ vérifiant $\tau^{*} \varpi=0$ et posons $\tau^{j}=y^{j} \circ \tau, \tau_{i}^{j}=p_{i}^{j} \circ \tau(i=1,2, \cdots, n ; j=1,2, \cdots, m)$. Alors, d'après (1.3), $\tau^{*} \widetilde{\sigma}=0$ entraîne $d \tau^{j}=\sum_{i=1}^{n} \tau_{i}^{j} d \bar{x}^{i}(j=1,2, \cdots, m)$ puisque $x^{i} \circ \tau=\bar{x}^{i}(i=1,2, \cdots, n)$ et par suite on a $\tau_{i}^{j}=\frac{\partial \tau^{j}}{\partial \bar{x}^{i}}$. Définissons une section $s$ de $\left.\pi\right|_{U}: U \rightarrow V$ par $x^{i} \circ s=\bar{x}^{i}, y^{j} \circ s=\tau^{j}(i=1,2, \cdots, n ; j$ $=1,2, \cdots, m)$. Les relations $\tau_{i}^{j}=\frac{\partial \tau^{j}}{\partial \bar{x}^{i}}$ signifient que $j^{1} s=\tau$. Donc on obtient le lemme suivant.

LEMME. Soit $\tau$ une section locale de $\alpha: J^{1}(M, \pi) \rightarrow N$ définie autour d'un point $x_{0} \in N$. Pour qu'il existe une section locale $s$ de $\pi$ définie autour $d u$ point $x_{0}$ telle que $j^{1} s=\tau$, il faut et il suffit que $\tau$ satisfasse à la condition $\tau^{*} \varpi=0$.

Proposition 1.3. Soient $R$ une équation différentielle du premier ordre $\operatorname{sur}(M, \pi, N)$ et $\omega$ la forme canonique sur $R$. Soit $\tau$ une section locale de $\alpha: R \rightarrow N$. Pour que la section locale $s=\beta \circ \tau$ de $\pi$ donne une solution de $R$, il faut et il suffit que $\tau$ satisfasse à la condition $\tau^{*} \omega=0$.

En effet, soient $\varpi$ la forme canonique sur $J^{1}(M, \pi)$ et $\iota: R \subseteq J^{1}(M, \pi)$ l'inclusion, «๐ $\tau$ est une section locale de $\alpha^{1}$. On a $(\iota \circ \tau)^{*} \varpi=\tau^{*} \circ \iota^{*} \varpi$ $=\tau^{*} \omega=0$ et par suite, d'après le lemme précédent, $j^{1} s=\iota \circ \tau$. Il en résulte que $s$ donne une solution de $R$.

\section{Pseudo-groupes laissant invariant les équations différentielles}

4 Dans ce chapitre, nous traiterons les pseudo-groupes qui laissent 
invariant les équations différentielles. Au lieu des pseudo-groupes, nous considérerons, au fond, ses algèbres de Lie, c'est-à-dire les faisceaux en algèbres de Lie.

Dans toute la suite de ce chapitre, toutes les variétés sont supposées connexes ; $\pi: M \rightarrow N$ désignera une fibration. Etant donnée une application locale $f$ d'une variété $M^{\prime}$ dans une autre $M^{\prime \prime}$, nous désignerons toujours par $U(f)$ le domaine de $f$ et par $V(f)$ l'image de $f$.

Soient $\phi$ un difféomorphisme local de $M$ dans $M$ et $X$ un champs de vecteurs local dans $M$.

DÉfinition 2.1. Nous disons que $\phi$ est $\pi$-projetable s'il existe un difféomorphisme local $f$ de $N$ dans $N$ tel que $\pi \circ \phi=f \circ \pi$. Ensuite, $X$ est dit $\pi$-projetable s'il existe un champs de vecteurs local $Y$ dans $N$ tel que $\pi_{*}\left(X_{y}\right)=Y_{\pi(y)}$ pour tout $y \in U(X)$. Pour simplifier les notations, écrivons $f=t(\phi)$.

Soit $\phi$ un difféomorphisme lolal $\pi$-projetable de $M$ dans $M$. Définissons un difféomorphisme local $p(\phi)$ de $J^{1}(M, \pi)$ dans $J^{1}(M, \pi)$ par la formule : $p(\phi)(z)=\phi_{*} \circ z \circ t(\phi)_{*}^{-1}$, pour tout $z \in \beta^{-1}(U(\phi))$, où $\beta$ désigne l'application but. On a $\alpha \circ p(\phi)=f \circ \alpha$ et $\beta \circ p(\phi)=\phi \circ \beta, \alpha$ étant l'application source. Considérons maintenant un champs de vecteurs local $\pi$-projetable $X$ dans $M$. $X$ est engendré par la famille à un paramètre de transformations locales dans $M,\left\{\phi_{t}\right\}$, et toute $\phi_{t}$ est $\pi$-projetable. Notons par $p(X)$ le champs de vecteurs local dans $J^{1}(M, \pi)$ engendré par la famille $\left\{p\left(\phi_{t}\right)\right\}$. Il est immédiat de démontrer la proposition suivante.

Proposition 2.1. Soient $\phi, \phi^{\prime}$ des difféomorphismes locaux et $\pi$ projetables de $M$ dans $M, X, Y$ des champs de vecteurs locaux et $\pi$ projetables dans $M$.

1) Si $V\left(\phi^{\prime}\right)=U(\phi)$, alors $p\left(\phi \circ \phi^{\prime}\right)=p(\phi) \circ p\left(\phi^{\prime}\right)$.

2) $\beta_{*}(p(X))=X$.

3) Si $U(\phi)=U(X)$, alors $\phi_{*} X$ est $\pi$-projetable et $p\left(\phi_{*} X\right)=p(\phi)_{*} p(X)$.

4) $[X, Y]$ est encore $\pi-p r o j e t a b l e$ et $p([X, Y])=[p(X), p(Y)]$.

Soit $R$ une équation différentielle du premier ordre sur $(M, \pi, N)$.

DÉfinition 2.2. Nous dirons qu'un difféomorphisme local $\pi$-projetable $\phi$ laisse $R$ invariant si $p(\phi)$ vérifie

$$
p(\phi)\left(R \cap \beta^{-1}(U(\phi))\right)=R \cap \beta^{-1}(V(\phi))
$$


et qu'un champs de vecteurs local $\pi$-projetable $X$ dans $M$ laisse $R$ invariant si $p(X)$ est un champs de vecteurs local tangent à $R$.

La proposition suivante résulte de la Proposition 2.1.

Proposition 2.2. Soient $\phi$ un difféomorphisme local $\pi$-projetable et $X, Y$ des champs de vecteurs locaux $\pi$-projetables. Supposons qu'ils laissent $R$ invariant.

i) Si $U(\phi)=U(X), \phi_{*} X$ laisse $R$ invariant,

ii) Si $U(X)=U(Y),[X, Y]$ laisse $R$ invariant.

EXEMPLe (voir §2, Exemple I): Soient $f$ une isométrie locale de $N$ dans $N$ et $\psi$ celle de $Q$ dans $Q$. Il est clair que $\phi=f \times \psi$ est un difféomorphisme local $\pi$-projetable de $N \times Q$ dans $N \times Q$ et que $p(\phi)(z)$ appartient à $R(g, \tilde{g})$ si et seulement si $z \in R(g, \tilde{g})$. Cela signifie que $\phi$ laisse $R(g, \tilde{g})$ invariant. Il en est de même pour l'exemple II.

5 Désignons par TM le faisceau des germes de champs de vecteurs locaux dans $M$ et par $\underline{X}_{y}$ le germe en $y \in M$ du champs de vecteurs local $X$ défini autour du point $y$. $\underline{T M}$ est muni de la structure de faisceau d'algèbre de Lie. Soit $\mathscr{L}$ un sous-faisceau d'espace vectoriel de $\underline{T M}$, et posons, pour tout $y \in M$,

$$
D(\mathscr{L})_{y}=\left\{X_{y} \in T_{y}(M) \mid \underline{X}_{y} \in \mathscr{L}_{y}\right\},
$$

où $\mathscr{L}_{y}$ désigne l'espace vectoriel de tous les germes en $y$ de $\mathscr{L}$. Indiquons par $\Gamma(U, \mathscr{L}), U$ étant un ouvert de $M$, l'ensemble de toutes les sections de $\mathscr{L}$ définies sur $U$; comme on sait, l'élément de $\Gamma(U, \mathscr{L})$ s'identifie canoniquement avec un champs de vecteurs défini sur $U$; indiquons finalement par $\mathscr{P}(\mathscr{L})$ le pseudo-groupe engendré par $\mathscr{L}$.

DÉFINITION 2.3. Un sous-faisceau d'espaces vectoriels, $\mathscr{L}$, de $\underline{T M}$ est dit un faisceau en algèbres de Lie si et seulement si $\mathscr{L}$ vérifie les conditions suivantes:

1) pour tous $X, Y \in \Gamma(U, \mathscr{L}),[X, Y] \in \Gamma(U, \mathscr{L})$,

2) $\phi \in \mathscr{P}(\mathscr{L})$ et $X \in \Gamma(U(\phi), \mathscr{L})$ implique $\phi_{*} X \in \Gamma(V(\phi), \mathscr{L})$,

3) $\mathscr{L}$ est défini par un système d'équations différentielles aux dérivées partielles d'ordre quelconque.

Lorsque $\mathscr{L}$ satisfait, en outre, à la condition

4) $\operatorname{dim} D(\mathscr{L})_{y}=$ constant, pour tout $y \in M$, $\mathscr{L}$ est dit régulier. En particulier, si $\mathscr{L}$ vérifie 
4) $D(\mathscr{L})_{y}=T_{y}(M)$, pour tout $y \in M$, $\mathscr{L}$ est appelé transitif.

A propos de cette définition, surtout de la condition 3), on peut voir les subtilités dans [11]. Dans toute la suite, $\mathscr{L}$ désigne un faisceau en algèbres de Lie de base $M$. Remarquons que si $\mathscr{L}$ est régulier, $D(\mathscr{L})$ forme une distribution involutive et la restriction de $\mathscr{L}$ à une feuille intégrale $\tilde{M}$ de $D(\mathscr{L})$ est un faisceau en algèbres de Lie transitif sur $\tilde{M}$.

DÉFINITION 2.4. $\mathscr{L}$ sera dit $\pi$-projetable si et seulement si toute section locale de $\mathscr{L}$ est $\pi$-projetable.

Soit $\mathscr{L} \pi$-projetable. Désignons par $\tilde{\pi}$ le morphisme de faisceaux défini par $\left.\tilde{\pi}\left(\underline{X}_{y}\right)=\underline{(\pi *}_{*}\right)_{\pi(y)} \in \underline{T M}$ pour tout $\underline{X}_{y} \in \mathscr{L}_{y}$. On a $\tilde{\pi}\left[Z, Z^{\prime}\right]$ $=\left[\tilde{\pi} Z, \tilde{\pi} Z^{\prime}\right]$ pour tous $Z, Z^{\prime} \in \mathscr{L}_{y}$. Posons $k(\mathscr{L})=\operatorname{Ker} \tilde{\pi} . \quad k(\mathscr{L})$ est considéré comme le faisceau des germes de champs de vecteurs de $\mathscr{L}$ tangents aux fibres de la fibration $\pi$. Il est clair que $k(\mathscr{L})_{y}$ est un idéal de $\mathscr{L}_{y}$.

Nous allons définir la filtration en $y\left\{\mathscr{L}_{y}^{r}\right\}_{r \in Z}$ comme suivant:

$$
\begin{aligned}
\mathscr{L}_{y}^{r}=\mathscr{L}_{y} \quad(r<0), \quad \mathscr{L}_{y}^{0}=\left\{\underline{X}_{y} \in \mathscr{L}_{y} \mid X_{y}=0\right\}, \\
\mathscr{L}_{y}^{r}=\left\{\underline{X}_{y} \in \mathscr{L}_{y} \mid j_{y}^{r}(X)=0\right\} \quad(r \geqq 1)
\end{aligned}
$$

et posons $k(\mathscr{L})_{y}^{r}=k(\mathscr{L})_{y} \cap \mathscr{L}_{y}^{r}(r \in \boldsymbol{Z})$. Il est facile de démontrer les relations: $\left[\mathscr{L}_{y}^{r}, \mathscr{L}_{y}^{s}\right] \subset \mathscr{L}_{y}^{r+s},\left[\mathscr{L}_{y}^{r}, k(\mathscr{L})_{y}^{s}\right] \subset k(\mathscr{L})_{y}^{r+s}(r, s \in \boldsymbol{Z})$; la première relation signifie que $\mathscr{L}_{y}^{r}$ est un idéal de $\mathscr{L}_{y}^{s}(r \geqq s \geqq 0)$.

Considérons l'algèbre de Lie $L=\lim _{\leftarrow} \mathscr{L}_{y} / \mathscr{L}_{y}^{r}$, appelée l'algèbre formelle de $\mathscr{L}$ en $y, k(L)=\lim _{\longleftarrow} k(\mathscr{L})_{y} / k(\mathscr{L})_{y}^{r}$ et $L^{0}=\lim _{\leftarrow} \mathscr{L}_{y}^{0} / \mathscr{L}_{y}^{r}$. $\quad k(L)$ peut être considéré comme un idéal de $L$ puisque $k(\mathscr{L})_{y}$ est un idéal de $\mathscr{L}_{y}$ et $k(\mathscr{L})_{y}^{r} \subset \mathscr{L}_{y}^{r}(r=1,2, \cdots)$. On a facilement $\left[L^{0}, L^{0}\right] \subset L^{0}$. Nous avons ainsi obtenu le triplet $\left(L, L^{0}, k(L)\right)$, que nous appellerons le $K$-système d'algèbres de Lie attachée à $\mathscr{L}$ en $y$.

Proposition 2.3. Soit $\mathscr{L} \pi$-projetable et supposons $\pi_{*}\left(D(\mathscr{L})_{y}\right)$ $=T_{x(y)}(N)$ pour tout $y \in M$. Soit $\left(L, L^{0}, k(L)\right)$ le $K$-système d'algèbres de Lie attaché à $\mathscr{L}$ en $y \in M$, et posons $V=L / L^{0}, W=k(L) / k(L) \cap L^{0}$. Alors $V$ s'identifie canoniquement avec l'espace vectoriel $D(\mathscr{L})_{y}$ et $V / W$ avec $T_{\pi(y)}(N)$.

En effet, on observe aisément que $L / L^{0}$ (resp. $k(L) / k(L) \cap L^{0}$ ) est isomorphe à $\mathscr{L}_{y} / \mathscr{L}_{y}^{0}\left(\operatorname{resp} . k(\mathscr{L})_{y} / k(\mathscr{L})_{y}^{0}\right)$ et par suite, $V$ (resp. $\left.W\right)$ s'identi- 
fie avec $D(\mathscr{L})_{y}$ (resp. $\left.D(\mathscr{L})_{y} / D(\mathscr{L})_{y} \cap \operatorname{Ker}\left(\pi_{*}\right)_{y}\right)$. Donc l'application linéaire surjective $\pi_{*}: D(\mathscr{L})_{y} \rightarrow T_{\pi(y)}(N)$ entraîne l'isomorphisme $\tilde{\pi}_{*}: V / W$ $\rightarrow T_{\pi(y)}(N)$.

Définissons une série de sous-espaces vectoriels $L \supset L^{0} \supset L^{1} \supset L^{2} \ldots$ par induction:

$$
\begin{array}{cc}
L^{1} & =\left\{X \in L^{0} \mid[X, L] \subset L^{0}\right\} \\
\vdots & \vdots \\
L^{r}= & \left\{X \in L^{r-1} \mid[X, L] \subset L^{r-1}\right\}
\end{array}
$$

Lorsque $\mathscr{L}$ est transitif, il se trouve que $L^{p}$ coïncide avec $\underset{r}{\lim _{r}} \mathscr{L}_{y}^{p} / \mathscr{L}_{y}^{p+r}$ (cf. [11]). Lorsque $\mathscr{L}$ est régulier, la filtration ci-dessus a quelque relation à celle de l'algèbre formelle du faisceau réstreint (voir [7]).

6 Soit $L$ une $R$-algèbre de Lie de dimension finie ou infinie et soit $L^{0}$ une sous-algèbre de Lie de $L$.

DÉfinition 2.5. Etant donné un idéal $K$ de $L$, nous dirons que $\left(L, L^{0}, K\right)$ est un $K$-système d'algèbres de Lie si $\operatorname{dim} L / L^{0}<\infty$ est vérifié.

Soit $\left(L, L^{0}, K\right)$ un $K$-système d'algèbres de Lie, et posons $L^{1}$ $=\left\{X \in L^{0} \mid[X, L] \subset L^{0}\right\}$ et pour simplifier les notations, écrivons $V=L / L^{0}$, $W=K / K \cap L^{0}, \mathrm{~g}=L^{0} / L^{1} . \quad$ On a immédiatement $\left[L, L^{1}\right] \subset L^{0},\left[L^{0}, L^{1}\right] \subset L^{1}$ et $\left[L^{1}, L^{1}\right] \subset L^{1}$. Il est facile de démontrer de cela que $\mathrm{g}$ est muni de la structure d'algèbre de Lie, appelée l'algèbre d'isotropie linéaire de $\left(L, L^{0}\right)$, et que $g$ est considérée comme une sous-algèbre de Lie de $\mathfrak{g l}(V)$ par la représentation fidèle $f: \mathfrak{g} \rightarrow \mathfrak{g l}(V)$ définie par $f\left(p_{0}\left(X_{0}\right)\right)(p(X))$ $=p\left(\left[X_{0}, X\right]\right)$, pour tous $X \in L, X_{0} \in L^{0}$, où $p_{0}$ (resp. $p$ ) désigne la projection canonique $L^{0} \rightarrow \mathfrak{g}$ (resp. $L \rightarrow V$ ). $W$ s'identifie avec un sous-espace vectoriel de $V$ et $[L, K] \subset K$ signifie que $g$ laisse $W$ invariant.

Indiquons par $\operatorname{gr}(V, W)$ la plus grande sous-algèbre de Lie qui laisse $W$ invariant. Soit $\sigma: V / W \rightarrow V$ un relèvement, c'est-à-dire une application linéaire vérifiant $\tilde{\pi} \circ \sigma=\mathrm{id}, \tilde{\pi}$ étant la projection $V \rightarrow V / W$. Définissons l'application linéaire surjective $\phi_{o}: \operatorname{gr}(V, W) \rightarrow \operatorname{Hom}(V / W, W)$ par la formule: $\phi_{\sigma}(X)(\xi)=X \circ \sigma(\xi)-\sigma \circ \tilde{\pi} \circ X \circ \sigma(\xi)$ pour tous $X \in \mathfrak{g r}(V, W)$, $\xi \in V / W$.

LEMME 2.1. Si $X \in \operatorname{Ker} \phi_{o}$,

i) $X^{n} \circ \sigma=\sigma \circ \tilde{\pi} \circ X^{n} \circ \sigma(n=1,2, \cdots)$, 
ii) $(\exp (s X)) \circ \sigma=\sigma \circ \tilde{\pi}(\exp (s X)) \circ \sigma$ pour tout $s \in R$, où $\exp (X)$ $=\sum_{n=0}^{\infty} \frac{X^{n}}{n !}$.

En effet, i) sera évident pour $n=0$ et $X \in \operatorname{Ker} \phi_{\sigma}$ signifie par définition que $X \circ \sigma=\sigma \circ \tilde{\pi} \circ X \circ \sigma$. Supposons que i) soit valable pour $n(n \geqq 1)$. Alors

$$
X^{n+1} \circ \sigma=X \circ \sigma \circ \tilde{\pi} \circ X^{n} \circ \sigma=\sigma \circ \tilde{\pi} \circ X \circ \sigma \circ \tilde{\pi} \circ X^{n} \circ \sigma=\sigma \circ \tilde{\pi} \circ X^{n+1} \circ \sigma .
$$

Donc i) est établi par induction. ii) résulte immédiatement de i).

Pour une sous-algèbre de Lie $g$ de $\operatorname{gr}(V, W)$, nous définissons les deux applications linéaires

$$
\begin{gathered}
\partial: \operatorname{Hom}(V, \mathfrak{g}) \rightarrow \operatorname{Hom}(V \wedge V, V) \\
\delta_{\sigma}: \operatorname{Hom}(V / W, \mathfrak{g}) \rightarrow \operatorname{Hom}(V / W \wedge V / W, W)
\end{gathered}
$$

par les formules:

$$
\begin{gathered}
(\partial S)(u \wedge v)=S(u) v-S(v) u \quad(u, v \in V), \\
\left(\delta_{\sigma} T\right)(\xi \wedge \eta)=\phi_{\sigma}(T(\xi))(\eta)-\phi_{\sigma}(T(\eta))(\xi) \quad(\xi, \eta \in V / W),
\end{gathered}
$$

pour tout $S \in \operatorname{Hom}(V, \mathfrak{g})$ et tout $T \in \operatorname{Hom}(V / W, \mathfrak{g})$. En outre, nous définissons les deux applications linéaires

$$
\begin{aligned}
\sigma^{*} & : \operatorname{Hom}(V, \mathfrak{g}) \rightarrow \operatorname{Hom}(V / W, \mathfrak{g}), \\
\hat{\sigma} & : \operatorname{Hom}(V \wedge V, V) \rightarrow \operatorname{Hom}(V / W \wedge V / W, W)
\end{aligned}
$$

par les formules: $\sigma^{*}(S)=S \circ \sigma$,

$$
(\hat{\sigma}(A))(\xi \wedge \eta)=A(\sigma(\xi) \wedge \sigma(\eta))-\sigma \circ \tilde{\pi} \circ A(\sigma(\xi) \wedge \sigma(\eta))
$$

$(\xi, \eta \in V / W)$ pour tout $S \in \operatorname{Hom}(V, \mathfrak{g})$ et tout $A \in \operatorname{Hom}(V \wedge V, V)$.

Cela étant, on a aisément $\hat{\sigma} \circ \partial=\delta_{\sigma} \circ \sigma^{*}$. Par conséquent, on obtient l'application linéaire $\bar{\sigma}: \operatorname{Hom}(V \wedge V, V) / \operatorname{Im} \partial \rightarrow \operatorname{Hom}(V / W \wedge V / W, W) / \operatorname{Im} \delta_{\sigma}$ définie pár

$$
\bar{\sigma}\left(\pi_{\partial}(A)\right)=\pi_{\sigma}(\hat{\sigma}(A)) \quad(A \in \operatorname{Hom}(V \wedge V, V)),
$$

où $\pi_{\partial}$ (resp. $\left.\pi_{\sigma}\right)$ désigne la projection canonique de Hom $(V \wedge V, V)$ sur $\operatorname{Hom}(V \wedge V, V) / \operatorname{Im} \partial$ (resp. de $\operatorname{Hom}(V / W \wedge V / W, W)$ sur $\operatorname{Hom}(V / W$ $\left.\wedge V / W, W) / \operatorname{Im} \delta_{\sigma}\right)$.

Conșidérons encore un $K$-système d'algèbres de Lie $\left(L, L^{0}, K\right)$. Soit 
$p: L \rightarrow V=L / L^{0}$ la projection canonique, prenons un relèvement $\tau$ de $p$. Pour ce relèvement, nous pouvons déterminer l'élément $C_{\imath} \in \operatorname{Hom}(V \wedge V, V)$ par $C_{\tau}(u \wedge v)=p([\tau(u), \tau(v)])(u, v \in V)$. Il se trouve que l'élément $C$ $=\pi_{\partial}\left(C_{\tau}\right)$ de Hom $(V \wedge V, V) / \operatorname{Im} \partial$ ne dépend pas du choix de $\tau$. L'élément $C$ ainsi obtenu est appelé la constante structurale de $\left(L, L^{0}\right)$.

DÉfinition 2.6. Soit $\left(L, L^{0}, K\right)$ un $K$-système d'algèbres de Lie et soit $C$ la constante structurale de $\left(L, L^{0}\right)$. L'élément $C_{\sigma}$ de $\operatorname{Hom}(V / W$ $\wedge V / W, W) / \operatorname{Im} \delta_{\sigma}$ défini par $\bar{\sigma}(C)=C_{o}$ sera dit la constante structurale partielle par rapport à $\sigma$.

7 Soit $\pi: M \rightarrow N$ une fibration et soit $\mathscr{L}$ un faisceau en algèbres de Lie $\pi$-projetable.

DÉFINITION 2.7. Une équation différentielle du premier ordre $R$ sur $(M, \pi, N)$ sera dit invariant par $\mathscr{L}$ si et seulement si toute section locale de $\mathscr{L}$ laisse $R$ invariant (cf. Définition 2.2).

Proposition 2.4. Soit $R$ une équation différentielle du premier ordre $\operatorname{sur}(M, \pi, N)$. Alors le faisceau des germes de tous les champs de vecteurs locaux et $\pi$-projetables dans $M$ laissant $R$ invariant est un faisceau en algèbres de Lie.

En effet, les conditions 1) et 2) de la Définition 2.3 résulte de la Proposition 2.2. La condition de tangence de $p(X)$ à $R, X$ étant un champs de vecteurs local et $\pi$-projetable dans $M$, est représentée par un système d'équations différentielles. On a donc 3) de la Définition 2.3 .

Un problème que nous voulons traiter principalement dans ce chapitre est celui d'existence des équations différentielles invariantes par un faisceau en algèbres de Lie. Soit $\mathscr{L} \pi$-projetable, et posons

$$
D^{1}(\mathscr{L})_{z}=\left\{p(X)_{z} \in T_{z}\left(J^{1}(M, \pi)\right) \mid \underline{X}_{y} \in \mathscr{L}_{y}(y=\beta(z))\right\} .
$$

En général, $D^{1}(\mathscr{L})$ est une distribution avec singularités. On doit passer la situation aux $G$-structures.

8 Soit $F(M)$ le fibré principal des repères de $M$ avec la fibration canonique $\widetilde{\varpi}: F(M) \rightarrow M . \quad F(M)$ est considéré comme l'ensemble de toutes les applications linéaires isomorphes de la forme suivante:

$$
u: V_{0} \rightarrow T_{x}(M) \quad(x \in M),
$$


où $V_{0}=R^{n+m}(n+m=\operatorname{dim} M)$. Soit $G$ un sous-groupe de Lie de $G L(n+m, \boldsymbol{R})$ et soit $W_{0}=\boldsymbol{R}^{m}$ le sous-espace vectoriel canonique de $V_{0}$.

DÉfinition 2.8. Nous dirons qu'une $G$-structure $B$ sur $M$ est $\pi$ projetable si et seulement si $u\left(W_{0}\right)=\operatorname{Ker}\left(\pi_{*}\right)_{y}(y=\varpi(u))$ est vérifié pour tout $u \in B$. Par suite, il faut que le groupe $G$ satisfasse à la condition: $g\left(W_{0}\right)=W_{0}$, pour tout $g \in G$.

Posons $P(\pi)=\left\{u \in F(M) \mid u\left(W_{0}\right)=\operatorname{Ker}\left(\pi_{*}\right)_{\approx(u)}\right\}$. Il est évident que $P(\pi)$ est une $G$-structure $\pi$-projetable, de base $M$, du groupe structural $G L\left(V_{0}, W_{0}\right)=\left\{g \in G L\left(V_{0}\right) \mid g\left(W_{0}\right)=W_{0}\right\}$. Etant donné un difféomorphisme local et $\pi$-projetable $\phi$ de $M$ dans $M$, nous pouvons définir un difféomorphisme local $\tilde{\phi}$ de $P(\pi)$ dans $P(\pi)$ par $\tilde{\phi}(u)=\phi_{*} \circ u(u \in P(\pi)$ avec $\widetilde{\sigma}(u) \in U(\phi))$. En conséquence, si $X$ est un champs de vecteurs local et $\pi$-projetable, $X$ est remonté à $P(\pi)$; notons le par $\tilde{X}$. Soit $\mathscr{L}$ transitif et $\pi$-projetable, et indiquons par $\mathscr{B}(\mathscr{L})$ le faisceau en algèbres de Lie déterminé par $\mathscr{L}$, i.e.,

$$
\mathscr{B}(\mathscr{L})_{u}=\left\{\underline{\tilde{X}}_{u} \in \underline{T(P(\pi))} \mid \underline{X}_{y} \in \mathscr{L}_{y}(y=\varpi(u))\right\} .
$$

Remarquons encore que toutes les variétés soient supposées connexes. Alors nous pouvons retrouver la proposition suivante (voir [11] Chap. II § 2.4).

Proposition 2.5. On a $\operatorname{dim} D(\mathscr{B}(\mathscr{L}))=\operatorname{dim} M+\operatorname{dim} \mathfrak{g}$, où $\mathfrak{g}$ désigne l'algèbre d'isotropie linéaire de $\mathscr{L}$. Soit $B_{u}$ une feuille intégrale de $D\left(\mathscr{B}(\mathscr{L})\right.$ ) passant par un point $u \in P(\pi)$. Alors $B_{u} \subset P(\pi)$ est une $G$ structure sur $M$, appelée une G-structure associée à $\mathscr{L}$, et l'algèbre de Lie du groupe structural de $B_{u}$ s'identifie avec g par le repère $u$. Pour un autre point $u^{\prime} \in P(\pi)$, il existe un élément $g \in G L\left(V_{0}, W_{0}\right)$ tel que $R_{g}\left(B_{u}\right)=B_{u^{\prime}}$.

En vertu de cette proposition, nous pouvons passer toute situation aux $G$-structures (transitifs).

Pour chaque $u \in P(\pi)$, indiquons par $t(u)$ l'application linéaire isomorphe de $V_{0} / W_{0}$ sur $T_{x}(N)(x=\pi \circ \varpi \circ(u))$ définie par la formule:

$$
\pi_{*} \circ u=t(u) \circ \tilde{\pi}_{0},
$$

$\tilde{\pi}_{0}$ étant la projection $V_{0} \rightarrow V_{0} / W_{0}$, et pour un relèvement $\sigma$ de $\tilde{\pi}_{0}$ définissons l'application $\Phi_{\sigma}: P(\pi) \rightarrow J^{1}(M, \pi)$ par la formule: 


$$
\Phi_{\sigma}(u)=u \circ \sigma \circ t(u)^{-1} .
$$

On a, pour l'application but $\beta: J^{1}(M, \pi) \rightarrow M$,

$$
\beta \circ \Phi_{\sigma}=\varpi .
$$

On peut observer que $\Phi_{\sigma}$ est différentiable.

Proposition 2.6. Soit $\phi$ un difféomorphisme (local) $\pi$-projetable de $M$ sur $M$ et soit $X$ un champs de vecteurs (local) $\pi$-projetable dans $M$. Alors les relations suivantes sont vérifiées.

1) $t(\tilde{\phi}(u))=t(\phi)_{*} \circ t(u)$,

2) $p(\phi) \circ \Phi_{\sigma}=\Phi_{\sigma} \circ \tilde{\phi}$,

3) $\left(\Phi_{o}\right)_{*} \tilde{X}=p(X)$.

Pour simplifier les notations, désignons par ã l'algèbre de Lie $\operatorname{gl}\left(V_{0}, W_{0}\right)$ et par $k^{\circ}(\tilde{\mathrm{g}})$ l'algèbre de Lie $\operatorname{Ker} \phi_{\sigma}$ (cf. $\left.\S 6\right)$. Pour tout $A \in \tilde{\mathrm{g}}$, $A^{*}$ désigne le champs de vecteurs $\varpi$-vertical sur $P(\pi)$ déterminé par l'opération de $G L\left(V_{0}, W_{0}\right)$ dans $P(\pi)$. Posons pour $u \in P(\pi)$

$$
k^{\circ}(\tilde{\mathfrak{g}})_{u}^{*}=\left\{A_{u}^{*} \in T_{u}(P(\pi)) \mid A \in k^{\circ}(\tilde{\mathfrak{g}})\right\} .
$$

LEMME 2.2. Si $A \in k^{\circ}(\tilde{\mathfrak{g}}), \Phi_{\sigma}(u \cdot \exp (s A))=\Phi_{\sigma}(u)$ pour tout $u \in P(\pi)$ et tout $s \in \boldsymbol{R}$.

En effet, pour $g \in G L\left(V_{0}, W_{0}\right)$ indiquons par $t(g)$ l'application linéaire isomorphe de $V_{0} / W_{0}$ sur lui-même définie par $t(g) \circ \pi_{0}=\tilde{\pi}_{0} \circ g$. On a aisément, pour tout $u \in P(\pi)$ et tout $g \in G L\left(V_{0}, W_{0}\right), t(u \circ g)=t(u) \circ t(g)$. Donc la condition du lemme est équivalente à $(\exp (s A)) \circ \sigma \circ t(\exp (s A))^{-1}$ $=\sigma$ pour tout $s \in R$ et par suite, à la condition $(\exp (s A)) \circ \sigma=\sigma \circ \tilde{\pi}_{0}$ $\circ(\exp (s A)) \circ \sigma$ pour tout $s \in R$. Alors ce lemme résulte du Lemme 2.1.

Proposition 2.7. $\operatorname{Ker}\left(\left(\Phi_{\sigma}\right)_{*}\right)_{u}=k^{\sigma}(\tilde{\mathfrak{g}})_{u}^{*}$ pour tout $u \in P(\pi)$.

Démonstration. La relation $k^{\sigma}(\tilde{\mathfrak{g}})_{u}^{*} \subset \operatorname{Ker}\left(\left(\Phi_{\sigma}\right)_{*}\right)_{u}$ résulte du Lemme 2.2. On peut démontrer que, pour tout $v \in T_{z}\left(J^{1}(M, \pi)\right)$, il existe un champs de vecteurs local et $\pi$-projetable $X$ dans $M$ tel que $p(X)_{z}=v$. Ce fait et 3) de la Proposition 2.6 signifient que $\Phi_{\sigma}$ possède le rang maximum sur $P(\pi)$. On a donc

$$
\begin{aligned}
\operatorname{dim}\left(\operatorname{Ker}\left(\left(\Phi_{\sigma}\right)_{*}\right)_{u}\right) & =\operatorname{dim} P(\pi)-\operatorname{dim} J^{1}(M, \pi) \\
& =\left(\operatorname{dim} M+\operatorname{dim} \mathfrak{g l}\left(V_{0}, W_{0}\right)\right)
\end{aligned}
$$




$$
\begin{aligned}
& -\left(\operatorname{dim} M+\operatorname{dim}\left(\operatorname{Hom}\left(V_{0} / W_{0}, W_{0}\right)\right)\right) \\
= & \operatorname{dim} \mathfrak{g l}\left(V_{0}, W_{0}\right)-\operatorname{dim}\left(\operatorname{Hom}\left(V_{0} / W_{0}, W_{0}\right)\right) \\
= & \operatorname{dim}\left(k^{\sigma}(\tilde{g})\right) .
\end{aligned}
$$

Comme la correspondance $\tilde{\mathrm{g}} \ni A \mapsto A_{u}^{*} \in T_{u}(P(\pi))$ est injective, on obtient $\operatorname{dim}\left(\operatorname{Ker}\left(\left(\Phi_{\sigma}\right)_{*}\right)_{u}\right)=\operatorname{dim} k^{\sigma}(\tilde{\mathfrak{g}})_{u}^{*}$. Il en résulte que $\operatorname{Ker}\left(\left(\Phi_{\sigma}\right)_{*}\right)_{u}$ coïncide avec $k^{\sigma}(\tilde{\mathfrak{g}})_{u}^{*}$. c.q.f.d.

Soit $B$ une $G$-structure $\pi$-projetable sur $M$ et soit $R$ une équation différentielle du premier ordre $\operatorname{sur}(M, \pi, N)$.

DÉFInItion 2.9. Nous dirons que $R$ est invariante par $B$ si et seulement si, pour tout $z \in R$, il existe un élément $u \in B$ et un relèvement $\sigma$ de $\tilde{\pi}_{0}: V_{0} \rightarrow V_{0} / W_{0}$ tels que
i) $\Phi_{\sigma}(B) \subset R$
ii) $\Phi_{\sigma}(u)=z$.

Proposition 2.8. Soit $\mathscr{L}$ transitif et $\pi$-projetable, de base $M$, et soit $R$ invariante par $\mathscr{L}$. Soit $B$ une G-structure associée à $\mathscr{L}$. Alors $R$ est invariante par $B$.

En effet, soit $z \in R$, et prenons un élément $u \in B$ vérifiant $\varpi(u)=\beta(z)$ et posons $\sigma=u^{-1} \circ z \circ t(u): V_{0} / W_{0} \rightarrow V_{0}$. Il est évident que $\sigma$ est un relèvement de $\tilde{\pi}_{0}$ et que $\Phi_{\sigma}(u)=z$. Comme $B$ est une feuille intégrale de $D\left(\mathscr{B}(\mathscr{L})\right.$ ) passant par un point, $\left.\mathscr{B}(\mathscr{L})\right|_{B}$ est transitif. $\mathscr{P}\left(\left.\mathscr{B}(\mathscr{L})\right|_{B}\right)$ est aussi transitif car $B$ est connexe. Chaque élément de $\mathscr{P}(\mathscr{B}(\mathscr{L})$ ) est de la forme $\tilde{\phi}, \phi \in \mathscr{P}(\mathscr{L})$ et par suite, pour tout $u^{\prime} \in B$, il existe un élément $\phi \in \mathscr{P}(\mathscr{L})$ tel que $\tilde{\phi}(u)=u^{\prime}$. Donc on a d'après la Proposition 2.6

$$
\Phi_{\sigma}\left(u^{\prime}\right)=\Phi_{\sigma}(\tilde{\phi}(u))=p(\phi) \circ \Phi_{\sigma}(u)=p(\phi)(z) .
$$

Puisque $\phi$ laisse $R$ invariant, on a $\Phi_{\sigma}\left(u^{\prime}\right) \in R$. Ceci démontre la condition i) de la Définition 2.9.

Proposition 2.9. Soit $R$ invariant par $B$. Alors tous les automorphismes locaux de $B$ laissent $R$ invariant. En conséquence, $R$ est invariant par le faisceau en algèbres de Lie $\pi$-projetable déterminé par les automorphismes infinitésimaux de $B$.

En effet, soit $\phi$ un automorphisme local de $B$, i.e.,

$$
\tilde{\phi}\left(B \cap \widetilde{\varpi}^{-1}(U(\phi))\right)=B \cap \widetilde{\varpi}^{-1}(V(\phi)) .
$$

Prenons un élément arbitraire $z \in R \cap \beta^{-1}(U(\phi))$ et soit $u \in B$ et $\sigma$ un 
relèvement vérifiant la condition de la Définition 2.9. On a d'après la Proposition 2.6

$$
p(\phi)(z)=p(\phi)\left(\Phi_{\sigma}(u)\right)=\Phi_{\sigma}(\tilde{\phi}(u)) .
$$

Cela signifie que $p(\phi)(z) \in R \cap \beta^{-1}(V(\phi))$. Comme $\phi$ est un difféomorphisme de $U(\phi)$ sur $V(\phi)$, on obtient aussi la relation inverse.

THÉORÈme 2.1. Soit $B$ une G-structure $\pi$-projetable sur $M$ et soit $\sigma$ un relèvement de $\tilde{\pi}_{0}: V_{0} \rightarrow V_{0} / W_{0}$. Alors $R=\Phi_{\sigma}(B)$ est une équation différentielle invariante par $B$.

LEMME 2.3. Pour tout relèvement $\sigma$ de $\tilde{\pi}_{0}$, l'application différentiable $\left.\Phi_{\sigma}\right|_{B}: B \rightarrow J^{1}(M, \pi)$ possède le rang constant sur $B$.

Si ce lemme est établi, nous pouvons démontrer le théorème à l'aide $d u$ théorème de fonction implicite.

Démonstration du Lemme 2.3. Soit g l'algèbre de Lie du groupe structural de $B$, et posons $k^{\sigma}(\mathfrak{g})=\operatorname{Ker} \phi_{o} \cap \mathfrak{g}$. Puisque

$$
\begin{aligned}
\operatorname{rang}_{u}\left(\left.\Phi_{\sigma}\right|_{B}\right) & =\operatorname{dim}\left(\operatorname{Im}\left(\left(\left(\left.\Phi_{\sigma}\right|_{B}\right)_{*}\right)_{u}\right)\right) \\
& =\operatorname{dim} B-\operatorname{dim}\left(\operatorname{Ker}\left(\left(\left(\left.\Phi_{o}\right|_{B}\right)_{*}\right)_{u}\right)\right)
\end{aligned}
$$

pour tout $u \in B$, il suffit de démontrer l'égalité $\operatorname{Ker}\left(\left(\left.\Phi_{\sigma}\right|_{B}\right)_{*}\right)_{u}=k^{\sigma}(\mathrm{g})_{u}^{*}$. La relation $k^{o}(\mathfrak{g})_{u}^{*} \subset \operatorname{Ker}\left(\left(\left.\Phi_{\sigma}\right|_{B}\right)_{*}\right)_{u}$ résulte immédiatement du Lemme 2.2. Soit $v \in \operatorname{Ker}\left(\left(\left.\Phi_{\sigma}\right|_{B}\right)_{*}\right)_{u}$, i.e., $v \in T_{u}(B) \cap \operatorname{Ker}\left(\left(\Phi_{\sigma}\right)_{*}\right)_{u}$. D'après la Proposition 2.7, il existe un élément $\tilde{A} \in \operatorname{Ker} \phi_{\sigma}$ tel que $\tilde{A}_{u}^{*}=v$. On a donc $\varpi_{*}(v)=0$. Par suite, il existe aussi un élément $A \in \mathfrak{g}$ tel que $A_{u}^{*}=v$. Comme l'application $\tilde{\mathrm{g}} \ni X \mapsto X_{u}^{*} \in T_{u}(P(\pi))$ est injective et $A, \tilde{A} \in \tilde{\mathrm{g}}$, on a $A=\tilde{A} \in \operatorname{Ker} \phi_{\sigma} \cap \mathrm{g}$. Ceci démontre que $v=A_{u}^{*} \in k^{o}(\mathfrak{g})_{u}^{*}$. c.q.f.d.

DÉFInItion 2.10. Nous dirons que $\mathscr{L}$ est $\pi$-transitif si $\mathscr{L}$ vérifie les conditions suivantes:

i) $\mathscr{L}$ est régulier et $\pi$-projetable,

ii) $\pi_{*}\left(D(\mathscr{L})_{y}\right)=T_{\pi(y)}(N)$ pour tout $y \in M$.

Remarquons que tous les faisceaux en algèbres de Lie transitifs et $\pi$ projetables sont $\pi$-transitifs.

THÉORÈmE 2.2. Soit $\mathscr{L} \pi$-transitif. Alors il existe toujours une équation différentielle du premier ordre sur $(M, \pi, N)$ invariante par $\mathscr{L}$. 
Démonstration. Comme $\mathscr{L}$ est régulier, nous pouvons considérer les feuilles intégrales de $D(\mathscr{L})$. Fixons un point $y_{0} \in M$ et indiquons par $M\left(y_{0}\right)$ la feuille intégrale de $D(\mathscr{L})$ passant par le point $y_{0}$ et par $\pi^{\prime}: M\left(y_{0}\right) \rightarrow N$ la réstriction de $\pi$ à $M\left(y_{0}\right)$. D'après la condition iii) de la Définition 2.10, il se trouve que $\pi^{\prime}$ est une fibration et que $M\left(y_{0}\right)$ est un sous-fibré de $M$ par rapport à $\pi$. Puisque le faisceau $\left.\mathscr{L}\right|_{M\left(y_{0}\right)}$ est transitif et $\pi^{\prime}$-projetable, d'après la Proposition 2.5, il existe une $G$ structure $\tilde{B}$ associée à $\left.\mathscr{L}\right|_{M\left(y_{0}\right)}$. En vertu du Théorème 2.1 et de la Proposition 2.9, il existe donc une équation différentielle du premier ordre $\tilde{R}$ sur $\left(M\left(y_{0}\right), \pi^{\prime}, N\right)$ invariante par $\left.\mathscr{L}\right|_{M\left(y_{0}\right)}$. Soit $\iota: M\left(y_{0}\right) \subseteq M$ l'inclusion, et considérons le morphisme de fibrés injectif $p(\iota): J^{1}\left(M\left(y_{0}\right), \pi^{\prime}\right)$ $\rightarrow J^{1}(M, \pi)$. Posons $R=p(\iota)(\tilde{R}) . \quad R$ est une équation différentielle du premier ordre sur $(M, \pi, N)$.

Nous allons démontrer finalement que tout élément de $\mathscr{P}(\mathscr{L})$ laisse $R$ invariant. Pour simplifier l'idée, supposons que $\phi \in \mathscr{P}(\mathscr{L})$ soit défini globalement sur $M$. Comme $\phi$ laisse $M\left(y_{0}\right)$ invariant, on peut considérer la réstriction de $\phi$ à $M\left(y_{0}\right), \psi$, i.e., $\phi \circ \iota=\iota \circ \psi$. Par suite, on a $p(\phi) p(\iota)$ $=p(\iota) p(\phi)$ et

$$
p(\phi)(R)=p(\phi) p(\iota)(\tilde{R})=p(\iota) p(\psi)(\tilde{R})=p(\iota)(\tilde{R})=R .
$$

Ceci démontre que $\phi$ laisse $R$ invariant. c.q.f.d.

S. Lie a étudié l'existence des équations différentielles invariantes par un pseudo-groupe continu donné en calculant des invariants différentiels de ce groupe (cf. [9]).

9 Finalement nous allons définir certaines fonctions structurales sur une $G$-structure $\pi$-projetable; elle sont en rapport étroit avec les constantes structurales attachées à un faisceau en algèbres de Lie transitif et $\pi$-projetable (cf. $\S 6$ ).

Soit $B$ une $G$-structure $\pi$-projetable sur $M$ et soit $\mathfrak{g}$ l'algèbre de Lie du groupe structural de $B$. Pour tout $A \in \mathfrak{g}$, désignons, comme précédent, par $A^{*}$ le champs de vecteurs sur $B$ déterminé par l'opération du groupe structural dans $B$. Considérons la fibration but $\beta: J^{1}(B, \varpi)$ $\rightarrow B$, $\varpi$ étant la projection de $B$ sur $M$; l'espace vectoriel $\operatorname{Hom}\left(V_{0}, \mathfrak{g}\right)$ peut opérer dans $J^{1}(B, \widetilde{\varpi})$ comme suivant:

$$
\left(S^{*} X\right)(v)=X(v)+S\left(u^{-1}(v)\right)_{u}^{*} \quad\left(v \in T_{y}(M), y=\varpi(u)\right),
$$


pour tout $S \in \operatorname{Hom}\left(V_{0}, \mathfrak{g}\right)$ et tout $X \in J^{1}(B, \varpi)$ avec $\beta(X)=u$. Il est facile de démontrer le

LeMme 2.4. Il existe, pour tous $X, X^{\prime} \in J^{1}(B, \varpi)$ avec $\beta(X)=\beta\left(X^{\prime}\right)$, un élément $S \in \operatorname{Hom}\left(V_{0}, \mathrm{~g}\right)$ tel que $X^{\prime}=S^{*} X$.

Soit $\theta$ la forme canonique sur $B$ définie par

$$
\theta_{u}(Z)=u^{-1}\left(\varpi_{*} Z\right) \quad\left(u \in B, Z \in T_{u}(B)\right) .
$$

$\theta$ possède les propriétés:

$$
\theta_{u}\left(A_{u}^{*}\right)=0, \quad i\left(A^{*}\right) d \theta=-A \theta
$$

pour tout $A \in \mathrm{g}$, où $i\left(A^{*}\right)$ désigne l'opération de produit intérieur par rapport à $A^{*}$. Définissons l'application $\bar{C}: J^{1}(B, \varpi) \rightarrow \operatorname{Hom}\left(V_{0} \wedge V_{0}, V_{0}\right)$ par la formule suivante:

$$
\bar{C}(X)(\xi \wedge \eta)=(d \theta)_{u}(X \cdot u(\xi) \wedge X \cdot u(\eta)) \quad(u=\beta(X))
$$

pour tous $\xi, \eta \in V_{0}$. On a aisément, pour tous $S \in \operatorname{Hom}\left(V_{0}, \mathfrak{g}\right)$ et $X$ $\in J^{1}(B, \varpi)$,

$$
\bar{C}\left(S^{*} X\right)=\bar{C}(X)-\partial S,
$$

où $\partial$ désigne l'opération définie par (2.1). En vertu du Lemme 2.4 et de (2.10), on obtient l'application $C: B \rightarrow \operatorname{Hom}\left(V_{0} \wedge V_{0}, V_{0}\right) / \operatorname{Im} \partial$ de sorte que

$$
\pi_{\partial} \circ \bar{C}=C \circ \beta
$$

soit établi, $\pi_{\partial}$ étant la projection canonique; c'est ce qu'on appelle la fonction structurale de $B$ (cf. [10], [11]).

Fixons un relèvement $\sigma$ de $\tilde{\pi}_{0}: V_{0} \rightarrow V_{0} / W_{0}$ et posons

$$
\Omega^{\sigma}=\theta-\sigma \circ \tilde{\pi}_{0} \circ \theta, \quad \Theta^{\sigma}=\sigma \circ \tilde{\pi}_{0} \circ \theta
$$

$\Omega^{\sigma}$ est la $W_{0}$-composante de $\theta$ et $\Theta^{\sigma}$ la $U_{0}$-composante de $\theta$, où $U_{0}$ $=\sigma\left(V_{0} / W_{0}\right)$.

LEMME 2.5. $i\left(A^{*}\right) d \Omega^{\sigma}=-A \circ \Omega^{\sigma}-\phi_{o}(A) \circ \tilde{\pi}_{0} \circ \theta$, pour tout $A \in \mathfrak{g}$.

En effet, d'après (2.9), il se trouve que $i\left(A^{*}\right) d \Omega^{o}$ est la $W_{0}$-composante de $-A \circ \theta$. On a par définition 


$$
\begin{aligned}
A \circ \theta & =A \circ \Omega^{\sigma}+A \circ \sigma \circ \tilde{\pi}_{0} \circ \theta \\
& =\left(A \circ \Omega^{\sigma}+\phi_{\sigma}(A) \circ \tilde{\pi}_{0} \circ \theta\right)+\left(\left(A-\phi_{\sigma}(A) \circ \tilde{\pi}_{0}\right) \circ \Theta^{\sigma}\right) \\
& =\left(A \circ \Omega^{\sigma}+\phi_{\sigma}(A) \circ \tilde{\pi}_{0} \circ \theta\right)+\sigma \circ \tilde{\pi}_{0} \circ A \circ \Theta^{\sigma} .
\end{aligned}
$$

Il est évident que $\sigma \circ \tilde{\pi}_{0} \circ A \circ \Theta^{\sigma}$ est une forme à valeurs dans $U_{0}$ et que $A \circ \Omega^{\sigma}+\phi_{\sigma}(A) \circ \tilde{\pi}_{0} \circ \theta$ dans $W_{0}$. Ceci démontre le lemme.

Soit $J^{1}(B, \pi \circ \varpi)$ la variété des 1 -jets de sections locales de $\pi \circ \varpi: B$ $\rightarrow N$, et indiquons par $R^{\sigma}(B)$ l'ensemble de tous les éléments vérifiant la condition:

$$
\theta_{u}(Y \circ t(u)(\xi))=\sigma(\xi) \quad\left(Y \in J^{1}(B, \pi \circ \varpi), u=\beta(Y)\right)
$$

pour tout $\xi \in V_{0} / W_{0}$.

Proposition 2.10. $R^{\sigma}(B)$ est un sous-fibré de $J^{1}(B, \pi \circ \varpi)$ par rapport à la fibration but $\beta: J^{1}(B, \pi \circ \varpi) \rightarrow B$.

En effet, la condition (2.12) est équivalente à $\Omega_{u}^{o}(Y \circ t(u)(\xi))=0 . \Omega^{\sigma}$ détermine un système de Pfaff sur $B, \Sigma$, et $R^{\sigma}(B)$ coïncide avec l'ensemble $R(\Sigma)$ défini dans $\S 2$ (cf. Exemple III). Il est facile de démontrer que $\Sigma$ vérifie la condition (C) dans $\S 2$. Cette proposition résulte donc de la Proposition 1.2.

L'espace vectoriel $\operatorname{Hom}\left(V_{0} / W_{0}, \mathfrak{g}\right)$ peut opérer dans $R^{\sigma}(B)$ comme suivant: soit $A \in \operatorname{Hom}\left(V_{0} / W_{0}, \mathfrak{g}\right)$ et soit $Y \in R^{o}(B)$,

$$
(A * Y)(v)=Y(v)+A\left(t(u)^{-1}(v)\right)_{u}^{*} \quad(u=\beta(Y)),
$$

pour tout $v \in T_{x}(N)(x=\pi \circ \varpi(u))$.

Proposition 2.11. Pour tous $Y, Y^{\prime} \in R^{\sigma}(B)$ avec $\beta(Y)=\beta\left(Y^{\prime}\right)$, il existe un élément $A \in \operatorname{Hom}\left(V_{0} / W_{0}, \mathfrak{g}\right)$ tel que $Y^{\prime}=A^{*} Y$. Si $A \in \operatorname{Hom}\left(V_{0} / W_{0}\right.$, g) vérifie, pour un élément $Y \in R^{\circ}(B), A^{*} Y=Y$, alors $A=0$.

En effet, il est facile de démontrer que

$$
\widetilde{\omega}_{*}\left(Y^{\prime} \circ t(u)(\xi)-Y \circ t(u)(\xi)\right)=0 \quad(u=\beta(Y))
$$

pour tout $\xi \in V_{0} / W_{0}$. Par suite, on n'a qu'à définir un élément $A$ de $\operatorname{Hom}\left(V_{0} / W_{0}, \mathfrak{g}\right) \operatorname{par} A(\xi)_{u}^{*}=Y^{\prime} \circ t(u)(\xi)-Y \circ t(u)(\xi)\left(\xi \in V_{0} / W_{0}\right)$. Si $Y^{\prime}=Y$ dans cette équation, on a $A(\xi)_{u}^{*}=0$ pour tout $\xi \in V_{0} / W_{0}$. Comme la correspondance $g \ni D \mapsto D_{u}^{*} \in T_{u}(B)$ est injective, on obtient $A=0$.

Pour simplifier les notations, écrivons $\tilde{Y}(\xi)=Y \circ t(u)(\xi)(u=\beta(Y)$, 
$\left.\xi \in V_{0} / W_{0}\right)$. Définissons d'abord l'application $\bar{C}_{o}: R^{\sigma}(B) \rightarrow \operatorname{Hom}\left(\left(V_{0} / W_{0}\right)\right.$ $\left.\wedge\left(V_{0} / W_{0}\right), W_{0}\right)$ par la formule:

$$
\bar{C}_{o}(Y)(\xi \wedge \eta)=\left(d \Omega^{o}\right)(\tilde{Y}(\xi) \wedge \tilde{Y}(\eta)),
$$

pour tout $Y \in R^{o}(B)$ et tout $\xi, \eta \in V_{0} / W_{0}$.

LEMME 2.6. $\bar{C}_{\sigma}\left(A^{*} Y\right)=\bar{C}_{\sigma}(Y)-\delta_{\sigma}(A)$, pour tous $Y \in R^{o}(B), \quad A$ $\in \operatorname{Hom}\left(V_{0} / W_{0}, \mathfrak{g}\right)$.

Pour $\xi, \eta \in V_{0} / W_{0}$, on a, d'après le Lemme 2.5, (2.2), (2.12) et (2.15),

$$
\begin{aligned}
\bar{C}_{o}\left(A^{*} Y\right)(\xi \wedge \eta)= & \left(d \Omega^{o}\right)\left(\left(\tilde{Y}(\xi)+A(\xi)_{u}^{*}\right) \wedge\left(\tilde{Y}(\eta)+A(\eta)_{u}^{*}\right)\right) \\
= & \left(d \Omega^{o}\right)(\tilde{Y}(\xi) \wedge \tilde{Y}(\eta))+\left(i\left(A(\xi)^{*}\right) d \Omega^{o}\right)(\tilde{Y}(\eta)) \\
& -\left(i\left(A(\eta)^{*} d \Omega^{\sigma}\right)(\tilde{Y}(\xi))+\left(i\left(A(\xi)^{*}\right) d \Omega^{o}\right)\left(A(\eta)_{u}^{*}\right)\right. \\
= & \bar{C}_{o}(Y)(\xi \wedge \eta)-\phi_{o}(A(\xi))(\eta)+\phi_{\sigma}(A(\eta))(\xi) \\
= & \bar{C}_{o}(Y)(\xi \wedge \eta)-\left(\delta_{\sigma} A\right)(\xi \wedge \eta) .
\end{aligned}
$$

Définissons l'application $C_{\sigma}: B \rightarrow \operatorname{Hom}\left(\left(V_{0} / W_{0}\right) \wedge\left(V_{0} / W_{0}\right), W_{0}\right) / \operatorname{Im} \delta_{\sigma}$ par la formule:

$$
C_{\sigma}(u)=\pi_{\sigma}\left(\bar{C}_{\sigma}(Y)\right),
$$

$Y$ étant un élément de $R^{\sigma}(B)$ vérifiant $u=\beta(Y)$. En vertu de la Proposition 2.11 et $\mathrm{du}$ Lemme 2.6, on voit facilement que $C_{\sigma}$ ne dépend pas du choix de $Y$. L'application $C_{o}$ sera dit la fonction structurale partielle par rapport à $\sigma$. Nous allons établir le rapport entre les deux fonctions $C$ et $C_{\sigma}$.

Proposition 2.12. $C_{\sigma}=\bar{\sigma} \cdot C$, où $\bar{\sigma}$ désigne l'application définie par (2.4).

Démonstration. Définissons l'application $\Psi: J^{1}(B, \varpi) \rightarrow J^{1}(B, \pi \circ \varpi)$ par $\Psi(X)=X \circ u \circ \sigma \circ t(u)^{-1}\left(X \in J^{1}(B, \widetilde{\varpi}), u=\beta(X)\right)$. On a $\varpi_{*} \circ \Psi(X) \circ t(u)=u \circ \sigma$ et donc $\Psi\left(J^{1}(B, \varpi)\right) \subset R^{\sigma}(B)$. Pour $\xi, \eta \in V_{0} / W_{0}$, on a

$$
\begin{aligned}
\bar{C}_{\sigma}(\Psi(X))(\xi \wedge \eta) & =\left(d \Omega^{\sigma}\right)((X \circ u \circ \sigma(\xi)) \wedge(X \circ u \circ \sigma(\eta))) \\
& =\left(d \theta-\sigma \circ \tilde{\pi}_{0} \circ d \theta\right)((X \circ u \circ \sigma(\xi)) \wedge(X \circ u \circ \sigma(\eta))) \\
& =\bar{C}(X)(\sigma(\xi) \wedge \sigma(\eta))-\sigma \circ \tilde{\pi}_{0} \circ \bar{C}(X)(\sigma(\xi) \wedge \sigma(\eta)) \\
& =(\hat{\sigma} \circ \bar{C}(X))(\xi \wedge \eta)
\end{aligned}
$$

et par suite, $\bar{C}_{\sigma} \circ \Psi=\hat{\sigma} \circ \bar{C}$, où $\hat{\sigma}$ est l'application définie par (2.3). En 
conséquence, on a, de plus,

$$
\bar{\sigma} \circ C \circ \beta=\bar{\sigma} \circ \pi_{\partial} \circ \bar{C}=\pi_{\sigma} \circ \hat{\sigma} \circ \bar{C}=\pi_{\sigma} \circ \bar{C}_{\sigma} \circ \Psi=C_{\sigma} \circ \beta \circ \Psi=C_{\sigma} \circ \beta .
$$

Il en résulte que $\bar{\sigma} \circ C=C_{\sigma}$. c.q.f.d.

Proposition 2.13. Pour qu'un point $u \in B$ annule $C_{o}$, il faut et $i l$ suffit qu'il existe un élément $Y \in R^{o}(B)$ tel que $\beta(Y)=u$ et $\bar{C}_{\sigma}(Y)=0$.

En effet, supposons que $u \in B$ annule $C_{\sigma}$ et prenons un élément $Y^{\prime} \in R^{\sigma}(B)$ avec $\beta\left(Y^{\prime}\right)=u$. On a $\bar{C}_{\sigma}\left(Y^{\prime}\right) \in \operatorname{Im} \delta_{\sigma}$. Il existe donc un élément $A \in \operatorname{Hom}\left(V_{0} / W_{0}, \mathfrak{g}\right)$ tel que $\bar{C}_{o}\left(Y^{\prime}\right)=\delta_{o}(A)$. Posons $Y=A * Y^{\prime}$. On a $\bar{C}_{o}(Y)=\bar{C}_{o}\left(Y^{\prime}\right)-\delta_{o}(A)=0$. La réciproque sera évidente.

Proposition 2.14. Si la fonction $C_{\sigma}$ est nulle sur $B$, il existe, pour tout $u_{0} \in B$, une section différentiable locale $\tilde{\tau}$ de $\beta: R^{\sigma}(B) \rightarrow B$ définie autour du point $u_{0}$ telle que $\bar{C}_{o}(\tilde{\tau}(u))=0$ pour tout $u \in U(\tilde{\tau})$.

En effet, prenons une section $\tau$ de $\beta: R^{\sigma}(B) \rightarrow B$. En fixant un sous-espace vectoriel $\mathscr{C}$ de $\operatorname{Hom}\left(V_{0} / W_{0}, \mathfrak{g}\right)$ vérifiant

$$
\operatorname{Hom}\left(V_{0} / W_{0}, \mathrm{~g}\right)=\left(\operatorname{Ker} \delta_{o}\right) \oplus \mathscr{C} \quad \text { (la somme directe) },
$$

nous définissons l'application linéaire isomorphe $\tilde{\delta}_{\sigma}: \mathscr{C} \rightarrow \operatorname{Im} \delta_{\sigma}$ par $\tilde{\delta}^{\sigma}$ $=\delta_{\sigma \mid \mathscr{\ell}^{\circ}}$ En outre, définissons l'application différentiable $\tilde{\tau}: U(\tau) \rightarrow R^{\sigma}(B)$ par $\tilde{\tau}(u)=\left(\tilde{\delta}_{o}\right)^{-1}\left(C_{o}(\tau(u)) * \tau(u)\right)(u \in U(\tau))$. On a

$$
\bar{C}_{\sigma}(\tilde{\tau}(u))=\bar{C}_{\sigma}(\tau(u))-\delta_{\sigma}\left(\tilde{\delta}_{\sigma}\right)^{-1}\left(\bar{C}_{\sigma}(\tau(u))=0 .\right.
$$

Considérons ensuite un faisceau $\mathscr{L}$ transitif et $\pi$-projetable sur $M$ et une $G$-structure associée à $\mathscr{L}, B$. Soit $\left(L, L^{0}, k(L)\right)$ le $K$-système d'algèbres de Lie attaché à $\mathscr{L}$ en $y \in M$.

Proposition 2.15. La fonction structurale partielle $C_{\sigma}$ sur $B$ est constante et coüncide avec la constante structurale partielle de $\left(L, L^{0}, k(L)\right)$ par rapport au relèvement $\sigma$.

En effet, soit $C$ la fonction structurale sur $B$ définie par (2.11). Il se trouve que $C$ est constante et que coïncide avec la constante structurale de $\left(L, L^{0}\right)$ (voir [11] Chap. II, § 2.10). Alors l'assertion résulte de la Définition 2.6 et de la Proposition 2.12 . 


\section{Théorèmes d'existence}

10 Dans ce chapitre, nous étudirons l'existence de solutions des équations différentielles invariantes par les pseudo-groupes. La première question est de trouver des conditions moyennant lesquelles les équations différentielles soient complètement réductibles (cf. Définition 1.3). Lorsque ceci est le cas, une telle équation différentielle est ramenée à certain système de Pfaff complètement intégrable. La deuxième question est de chercher des conditions moyennant lesquelles l'existence de solutions des équations différentielles soit ramenée au Théorème de Cartan-Kähler.

Dans ce chapitre, nous supposons encore que toutes les variétés considérées soient connexes et considérons une fibration $\pi: M \rightarrow N$ et désignons par $R$ une équation différentielle du premier ordre $\operatorname{sur}(M, \pi, N)$ et par $B$ une $G$-structure $\pi$-projetable et finalement indiquons par $\omega$ la forme canonique sur $R$ et par $\theta$ la forme canonique $\operatorname{sur} B$ (cf. (1.2) et (2.8)). Soit $V_{0}=\boldsymbol{R}^{n+m}$ et $W_{0}=\boldsymbol{R}^{m}(n=\operatorname{dim} N, n+m=\operatorname{dim} M)$. Prenons un relèvement $\sigma$ de $\tilde{\pi}_{0}: V_{0} \rightarrow V_{0} / W_{0}$ et posons $\Omega^{\sigma}=\theta-\sigma \circ \tilde{\pi}_{0} \circ \theta$.

LEMME 3.1. Soit $\sigma$ un relèvement de $\tilde{\pi}_{0}$ vérifiant $\Phi_{\sigma}(B) \subset R$. Alors on $a$

$$
u^{-1}\left(\Phi_{\sigma}^{*} \omega\right)_{u}\left(X_{u}\right)=\Omega_{u}^{\sigma}\left(X_{u}\right) \quad\left(u \in B, X_{u} \in T_{u}(B)\right) .
$$

En effet, on obtient, d'après les formules (1.2), (2.5), (2.6) et (2.8),

$$
\begin{aligned}
u^{-1}\left(\omega_{\Phi_{\sigma}(u)}\left(\left(\Phi_{\sigma}\right)_{*} X_{u}\right)\right) & =u^{-1}\left(\beta_{*}\left(\Phi_{\sigma}\right)_{*} X_{u}-u \circ \sigma \circ t(u)^{-1} \pi_{*} \beta_{*} \circ \Phi_{\sigma_{*}} X_{u}\right) \\
& =u^{-1}\left(\widetilde{\varpi}_{*} X_{u}-u \circ \sigma \circ \tilde{\pi}_{\sigma} \circ u^{-1} \circ \widetilde{\varpi}_{*} X_{u}\right) \\
& =\theta_{u}\left(X_{u}\right)-\sigma \circ \tilde{\pi}_{0} \circ \theta_{u}\left(X_{u}\right) \\
& =\Omega_{u}^{\sigma}\left(X_{u}\right) .
\end{aligned}
$$

Proposition 3.1. Soit $R$ invariante par $B$, et fixons un relèvement $\sigma$ tel que $\Phi_{\sigma}(B) \subset R$. Si $\rho$ est une section locale de $\pi \circ \widetilde{\sigma}: B \rightarrow N$ vérifiant $\rho^{*} \Omega^{o}=0$, alors $s=\widetilde{\sigma} \rho$ est une solution locale de $R$.

En effet, puisque $\tau=\Phi_{\sigma} \circ \rho$ est une section locale de $\alpha: R \rightarrow N$ et vérifie la relation $\beta \circ \tau=s$, il suffit de démontrer $\tau^{*} \omega=0$ (cf. Proposition 1.3). Pour tout $x \in U(\tau)$ et tout $X \in T_{x}(N)$, on a, d'après le lemme précédent,

$$
\begin{aligned}
\rho(x)^{-1}\left(\tau^{*} \omega\right)_{x}(X) & =\rho(x)^{-1}\left(\Phi_{\sigma}^{*} \omega\right)\left(\rho_{*} X\right) \\
& =\Omega^{o}\left(\rho_{*} X\right)=\left(\rho_{*} \Omega^{\sigma}\right)(X)=0
\end{aligned}
$$


et par suite, $\tau^{*} \omega=0$ puisque $\rho(x)^{-1}$ est un isomorphisme linéaire de $T_{s(x)}(M)$ sur $V_{0}$.

Cette proposition signifie que nous sommes ramenés à la recherche de l'équation différentielle du premier ordre $\operatorname{sur}(B, \pi \circ \varpi, N), R^{\circ}(B)$ (cf. $\S 2$. Exemple III, et Proposition 2.10). D'ailleurs, comme nous avons déjà assez de résultats sur les $G$-structures, l'objet $R^{\circ}(B)$ sera moins difficile à rechercher que $R$. D'abord, nous allons profiter de la notion géométrique «plate».

DÉFinition 3.1 (cf. [10], [11]). Etant donnée, en général, une $G$ structure $B$ sur $M$, nous disons que $B$ est plate si et seulement si, pour tout $y_{0} \in M$, il existe un système de coordonnées locales $\left(y^{1}, \cdots, y^{n+m}\right)$ $(n+m=\operatorname{dim} M)$ d'un voisinage ouvert $U$ de $y_{0}$ tel que $\lambda(y)\left(\boldsymbol{e}_{i}\right)=\left(\frac{\partial}{\partial y^{i}}\right)_{y}$ $(y \in U, i=1,2, \cdots, n+m)$ forme une section différentiable locale de $\varpi: B \rightarrow M$, où $\left\{\boldsymbol{e}_{1}, \cdots, \boldsymbol{e}_{n+m}\right\}$ est le système de base canonique de $V_{0}$.

THÉORÈmE 3.1. Soit $B$ une G-structure $\pi$-projetable sur $M$ et soit $R$ une équation différentielle du premier ordre sur $(M, \pi, N)$ invariante par $B$. Si $B$ est plate, alors $R$ est complètement réductible.

Démonstration. Soit $\left(U,\left(y^{1}, \cdots, y^{n+m}\right)\right)$ une carte de $M$ vérifiant la condition de la définition et soit $\lambda: U \rightarrow B$ la section canonique associée à la carte. En posant $\theta=\sum \theta^{i} e_{i}$, on a $\lambda^{*} \theta^{i}=d y^{i}(i=1,2, \cdots, n+m)$ puisque $\theta\left(\lambda_{*}\left(\frac{\partial}{\partial y^{i}}\right)_{y}\right)=\lambda(y)^{-1}\left(\frac{\partial}{\partial y^{i}}\right)_{y}=\boldsymbol{e}_{i}$. Ceci démontre que $d\left(\lambda^{*} \theta\right)=0$, par suite, pour un relèvement $\sigma$ vérifiant $\Phi_{\sigma}(B) \subset R$, on a $d\left(\lambda^{*} \Omega^{\sigma}\right)=d\left(\lambda^{*} \theta\right)$ $-\sigma \circ \tilde{\pi}_{0} \circ\left(d\left(\lambda^{*} \theta\right)\right)=0$. Posons $\lambda^{*} \Omega^{\sigma}=\sum_{i=1}^{m} \Omega^{i} \boldsymbol{e}_{n+i}$, alors le système de Pfaff $\sum=\left\langle\Omega^{1}, \cdots, \Omega^{m}\right\rangle$ sur $U$ devient complètement intégrable. Prenons une section arbitraire $s$ de $\pi$ vérifiant $s^{*} \Omega^{i}=0(i=1,2, \cdots, m)$. Posons $\rho=\lambda \circ s, \rho$ est une section locale de $\pi \circ \varpi: B \rightarrow N$ vérifiant $\rho^{*} \Omega^{\circ}=0$. D'après la Proposition 3.1, il se trouve que $s$ est encore une solution locale de $R$. Il en résulte que $R$ est complètement réductible. c.q.f.d.

Ensuite nous allons chercher autre condition sous laquelle les équations différentielles sont complètement réductibles. Supposons que $R$ soit une équation différentielle $d u$ premier ordre $\operatorname{sur}(M, \pi, N)$ invariante par $B$ et qu'un relèvement $\sigma$ de $\tilde{\pi}_{0}: V_{0} \rightarrow V_{0} / W_{0}$ satisfasse à $\Phi_{\sigma}(B) \subset R$. 
LEMme 3.2. Pour un vecteur $Z \in T_{u}(B)$, les deux conditions suivantes sont équivalentes:

i) $\Omega_{u}^{o}(Z)=0$,

ii) il existe un élément $Y \in R^{\circ}(B)$ avec $\beta(Y)=u$ et élément $A \in \mathfrak{g}$ tels que $Z=Y(v)+A_{u}^{*}\left(v=\pi_{*} \circ \widetilde{\varpi}_{*}(Z)\right)$.

En effet, supposant la condition i), on a

$$
\begin{aligned}
\Omega_{u}^{o}(Z) & =\theta_{u}(Z)-\sigma \circ \tilde{\pi}_{0} \circ \theta_{u}(Z) \\
& =\theta_{u}(Z)-\sigma \circ \tilde{\pi}_{0} \circ u^{-1} \circ{\varpi_{*}}(Z) \\
& =\theta_{u}(Z)-\sigma \circ t(u)^{-1} \circ \pi_{*} \circ{\varpi_{*}}_{*}(Z) \\
& =\theta_{u}(Z)-\sigma\left(t(u)^{-1}(v)\right)
\end{aligned}
$$

et par suite, $\theta_{u}(Z)=\sigma\left(t(u)^{-1}(v)\right)$. D'après la définition de $R^{\sigma}(B)$ et (2.12), on a aussi

$$
\begin{aligned}
\widetilde{\varpi}_{*}(Z-Y(v)) & =u \theta_{u}(Z-Y(v)) \\
& =u\left\{\theta_{u}(Z)-\sigma\left(t(u)^{-1}(v)\right)\right\} \\
& =0 .
\end{aligned}
$$

Ceci démontre ii). La réciproque sera évidente.

THÉOR亡̀me 3.2. Soit $B$ une G-structure $\pi$-projetable et soit $R$ une équation différentielle du premier ordre sur $(M, \pi, N)$ invariante par $B$. S'il existe un relèvement $\sigma$ de $\tilde{\pi}_{0}: V_{0} \rightarrow V_{0} / W_{0}$ tel que

1) $\Phi_{o}(B) \subset R$,

2) la fonction structurale partielle $C_{\sigma}$ est nulle sur $B$,

3) $\phi_{o}(\mathfrak{g})=\{0\}$, g étant l'algèbre de Lie du groupe structural de $B$, alors $R$ est complètement réductible.

Démonstration. D'après le Lemme 2.3 et la condition 1) ci-dessus, $R^{\prime}=\Phi_{\sigma}(B)$ est une équation différentielle vérifiant $R^{\prime} \subset R$. La condition 3) signifie que $k^{o}(\mathfrak{g})=\mathfrak{g}$, on a donc

$$
\begin{aligned}
\operatorname{dim} R^{\prime} & =\operatorname{dim} B-\operatorname{dim}\left(k^{\sigma}(\mathrm{g})\right) \\
& =\operatorname{dim} B-\operatorname{dim} \mathfrak{g}=\operatorname{dim} M .
\end{aligned}
$$

Il en résulte que la projection but $\beta^{\prime}: R^{\prime} \rightarrow M$ est un difféomorphisme et que la forme canonique $\omega^{\prime}$ sur $R^{\prime}$ détermine localement un système de Pfaff $\Sigma^{\prime}$ dans $M$. Il est clair que $R\left(\Sigma^{\prime}\right)=R^{\prime}$ (cf. Exemple III). Pour constater que le système $\Sigma$ est complètement intégrable, il suffit de 
démontrer, en vertu du Lemme 3.1 , que $\Omega^{\sigma}=0$ est un système de Pfaff complètement intégrable.

Considérons la distribution $D\left(\Omega^{o}\right)$ sur $B$ définie par

$$
D\left(\Omega^{\circ}\right)_{u}=\left\{Z \in T_{u}(B) \mid \Omega_{u}^{o}(Z)=0\right\} \text {. }
$$

D'après la Proposition 2.14, il existe, pour tout $u_{0} \in B$, une section locale $\tau$ de $\beta: R^{\sigma}(B) \rightarrow B$ définie autour du point $u_{0}$ telle que $\bar{C}_{\sigma}(\tau(u))=0$ pour tout $u \in U(\tau)$. Fixons une telle section locale $\tau$ et définissons pour tout $\xi \in V_{0} / W_{0}$ un champs de vecteurs $\widetilde{\tau(\xi)}$ sur $U(\tau)$ par la formule

$$
\widetilde{\tau(\xi)_{u}}=\tau(u)(t(u)(\xi)) \quad(u \in U(\tau)) .
$$

On a, d'après le Lemme $3.2, \Omega_{u}^{\sigma}\left(\widetilde{\tau(\xi)_{u}}\right)=0$ et $\Omega_{u}^{o}\left(A_{u}^{*}\right)=0$ pour tous $\xi \in V_{0} / W_{0}, A \in \mathrm{g}$ et tout $u \in U(\tau)$. Les champs de vecteurs $\widetilde{\tau(\xi)}\left(\xi \in V_{0} / W_{0}\right)$ et $A^{*}(A \in \mathrm{g})$ engendrennent la distribution puisque $\operatorname{dim} D\left(\Omega^{\sigma}\right)_{u}$ $=\operatorname{dim}\left(V_{0} / W_{0}\right)+\operatorname{dim} \mathrm{g}$. Par conséquent, on n'a qu'à démontrer les relations
i) $\left[A^{*}, B^{*}\right]_{u} \in D\left(\Omega^{\sigma}\right)_{u}$
( $A, B \in \mathrm{g})$,
ii) $\left[A^{*}, \widetilde{\tau(\xi)}\right]_{u} \in D\left(\Omega^{\sigma}\right)_{u}$
$\left(A \in \mathfrak{g}, \xi \in V_{0} / W_{0}\right)$,
iii) $\widetilde{[\tau(\xi)}, \widetilde{\tau(\eta)}]_{u} \in D\left(\Omega^{o}\right)_{u}$
$\left(\xi, \eta \in V_{0} / W_{0}\right)$.

Mais i) sera évident. On a

$$
\begin{aligned}
\Omega_{u}^{o}\left(\left[A^{*}, \widetilde{\tau(\xi)}\right]_{u}\right) & =-d \Omega^{o}\left(A_{u}^{*} \wedge \widetilde{\tau(\xi)_{u}}\right) \\
& =-\left(i\left(A^{*}\right) d \Omega^{\sigma}\right)\left(\widetilde{\tau(\xi)_{u}}\right) \\
& =-A \circ \Omega^{o}\left(\widetilde{\tau(\xi)_{u}}\right)+\phi_{o}(A) \circ \tilde{\pi}_{0} \circ \theta_{u}\left(\widetilde{\tau(\xi)_{u}}\right) \\
& =\phi_{o}(A)(\xi) .
\end{aligned}
$$

Ceci démontre ii) puisque $\phi_{o}(\mathrm{~g})=0$.

Ensuite on a

$$
\begin{aligned}
\Omega_{u}^{o}\left([\widetilde{\tau(\xi)}, \widetilde{\tau(\eta)}]_{u}\right) & \left.=-\left(d \Omega^{o}\right)_{u} \widetilde{\left(\tau(\xi)_{u}\right.} \wedge \widetilde{\tau(\eta)_{u}}\right) \\
& =-\bar{C}_{o}(\tau(u))(\xi \wedge \eta) .
\end{aligned}
$$

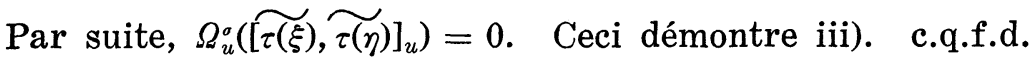

THÉORÈme 3.3. Soit $\mathscr{L}$ un faisceau en algèbres de Lie transitif et $\pi$-projetable et soit $R$ une équation différentielle du premier ordre sur $(M, \pi, N)$ invariante par $\mathscr{L}$. Soit $\left(L, L^{0}, k(L)\right)$ le K-système d'algèbres 
de Lie attaché à $\mathscr{L}$ en $y \in M$. S'il existe un point $z \in R$ tel que

i) $\beta(z)=y$,

ii) la fonction structurale partielle $C_{z}$ de $\left(L, L^{0}, k(L)\right)$ est nulle,

iii) $\phi_{z}(\mathrm{~g})=0, \mathfrak{g}$ étant l'algèbre d'isotropie linéaire de $\left(L, L^{0}, k(L)\right)$, alors $R$ est complètement réductible.

En effet, prenons un point $u \in P(\pi)$ avec $\widetilde{\sigma}(u)=y$ et posons $\sigma=u^{-1}$ $\circ z \circ t(u)$. Il est clair que $\sigma$ est un relèvement de $\tilde{\pi}_{0}: V_{0} \rightarrow V_{0} / W_{0}$ et que la $G$-structure $B$ associée à $\mathscr{L}$ passant par le point $u$ vérifie $\Phi_{\sigma}(B) \subset R$ (cf. Propositions 2.5 et 2.8). D'après la Proposition 2.3, $z$ s'identifie canoniquement avec un relèvement de $\tilde{\pi}: V \rightarrow V / W$, où $V$ (resp. $W$ ) désigne $L / L^{0}$ (resp. $k(L) / k(L) \cap L^{0}$ ). La fonction structurale partielle $C_{\sigma}$ sur $B$ coïncide par définition avec $C_{z}$ et par suite $C_{\sigma}=0$. Soit $g_{0}$ l'algèbre de Lie du groupe structural de $B$, alors $g$ est isomorphe à $g_{0}$ et $\phi_{z}(\mathfrak{g})$ est aussi isomorphe à $\phi_{\sigma}\left(\mathfrak{g}_{0}\right)$ par les isomorphismes construits par le repère $u$. Donc ce théorème résulte du Théorème 3.2.

11 Nous donnons ici quelques notions algébriques qui sont utilisées dans la suite.

Soient $V^{\prime}, V^{\prime \prime}$ des espaces vectoriels réels de dimension finie et $E$ un sous-espace vectoriel de $\operatorname{Hom}\left(V^{\prime}, V^{\prime \prime}\right)$. Nous définissons le prolongement algébrique d'ordre 1 de $E$ par

$$
E^{(1)}=\left\{X \in \operatorname{Hom}\left(V^{\prime}, E\right) \mid X(u)(v)=X(v)(u) \text { pour tous } u, v \in V^{\prime}\right\} .
$$

Soit $U^{r}$ un sous-espace vectoriel à $r$ dimensions de $V^{\prime}$, posons

$$
E\left(U^{r}\right)=\left\{f \in E \mid f(u)=0 \text { pour tout } u \in U^{r}\right\} .
$$

Fixons un sous-espace vectoriel à $r$ dimensions $U_{0}^{r}$ de $V^{\prime} ; U_{0}^{r}$ est dit générique si $U_{0}^{r}$ vérifie $\operatorname{dim} E\left(U_{0}^{r}\right) \leqq \operatorname{dim} E\left(U^{r}\right)$ pour tout sous-espace vectoriel à $r$ dimensions $U^{r}$ de $V^{\prime}$.

DÉFINITION 3.2. Un sous-espace vectoriel $E$ de Hom $\left(V^{\prime}, V^{\prime \prime}\right)$ est dit involutif si et seulement s'il existe une série de sous-espaces vectoriels génériques

$$
\{0\}=U^{0} \subset U^{1} \subset U^{2} \subset \cdots \subset U^{n^{\prime}}=V^{\prime} \quad\left(n^{\prime}=\operatorname{dim} V^{\prime}\right)
$$

telle que $\operatorname{dim} E^{(1)}=\sum_{r=0}^{n^{\prime}-1} \operatorname{dim} E\left(U^{r}\right)$.

Considérons maintenant un $K$-système d'algèbres de Lie $\left(L, L^{0}, K\right)$ et écrivons $V=L / L^{0}, W=K / K \cap L^{0}$. Soit $g$ l'algèbre d'isotropie 
linéaire de $\left(L, L^{0}, K\right) ; \mathfrak{g}$ est envisagée comme une sous-algèbre de Lie de $\operatorname{gr}(V, W)$ (voir $\S 6$ ). Prenons un relèvement $\sigma$ de $\tilde{\pi}: V \rightarrow V / W$ et considérons encore les applications linéaires $\phi_{\sigma}: \operatorname{gl}(V, W) \rightarrow \operatorname{Hom}(V / W, W)$ et $\delta_{\sigma}: \operatorname{Hom}(V / W, \mathfrak{g}) \rightarrow \operatorname{Hom}(V / W \wedge V / W, W)$ définies dans $\S 6$. Pour simplifier les notations, écrivons $k^{o}(\mathfrak{g})=\mathfrak{g} \cap \operatorname{Ker} \phi_{\sigma}, \mathfrak{g}(\sigma)=\phi_{o}(\mathrm{~g})$ et $I^{o}(\mathrm{~g})$ $=\operatorname{Ker} \delta_{\sigma}$.

LEMME 3.3. $\operatorname{dim} I^{o}(\mathfrak{g})=\operatorname{dim}(V / W) \times \operatorname{dim} k^{o}(\mathrm{~g})+\operatorname{dim} \mathfrak{g}(\sigma)^{(1)}$.

En effet, comme la suite

$$
0 \longrightarrow k^{\sigma}(\mathfrak{g}) \stackrel{\iota}{\longrightarrow} \mathfrak{g} \stackrel{\phi_{o}}{\longrightarrow} \mathfrak{g}(\sigma) \longrightarrow 0
$$

est exacte,

$$
0 \longrightarrow \operatorname{Hom}\left(V / W, k^{\sigma}(\mathrm{g})\right) \stackrel{\iota_{*}}{\longrightarrow} \operatorname{Hom}(V / W, \mathfrak{g}) \stackrel{\phi_{\sigma_{*}}}{\longrightarrow} \operatorname{Hom}(V / W, \mathrm{~g}(\sigma)) \longrightarrow 0
$$

est aussi exacte. Il est facile de cela de démontrer que la restriction de $\phi_{\sigma_{*}}$ à $I^{\sigma}(\mathfrak{g})$ possède l'image dans $\mathfrak{g}(\sigma)^{(1)}$ et en outre que la suite

$$
0 \longrightarrow \operatorname{Hom}\left(V / W, k^{o}(\mathfrak{g})\right) \stackrel{\iota_{*}}{\longrightarrow} I^{\sigma}(\mathfrak{g}) \stackrel{\phi_{o_{*}}}{\longrightarrow} \mathrm{g}(\sigma)^{(1)} \longrightarrow 0
$$

est exacte. Le lemme est ainsi établi.

Soit $U^{r}$ un sous-espace vectoriel à $r$ dimensions de $V / W$. Posons

$$
\begin{aligned}
\mathfrak{g}_{o}\left(U^{r}\right) & =\left\{A \in \mathfrak{g} \mid \phi_{\sigma}(A)(\eta)=0 \text { pour tout } \eta \in U^{r}\right\}, \\
\mathrm{g}\left(\sigma ; U^{r}\right) & =\left\{C \in \mathrm{g}(\sigma) \mid C(\eta)=0 \text { pour tout } \eta \in U^{r}\right\} .
\end{aligned}
$$

LEMME 3.4. $\operatorname{dim} \mathfrak{g}_{\sigma}\left(U^{r}\right)=\operatorname{dim} k^{o}(\mathfrak{g})+\operatorname{dim} \mathfrak{g}\left(\sigma ; U^{r}\right)$

En effet, la suite

$$
0 \longrightarrow k^{\sigma}(\mathrm{g}) \stackrel{\iota}{\longrightarrow} \mathrm{g}_{\sigma}\left(U^{r}\right) \stackrel{\phi_{\sigma}}{\longrightarrow} \mathrm{g}\left(\sigma ; U^{r}\right) \longrightarrow 0
$$

est exacte.

Proposition 3.2. Si $\mathrm{g}(\sigma)$ est involutif par rapport à une série de sous-espaces vectoriels génériques

$$
\{0\}=U^{0} \subset U^{1} \subset U^{2} \subset \cdots \subset U^{n}=V / W \quad(n=\operatorname{dim}(V / W)),
$$

alors

i) $\operatorname{dim} I^{o}(\mathfrak{g})=\sum_{r=0}^{n-1} \operatorname{dim} g_{o}\left(U^{r}\right)$,

ii) $\operatorname{dim} g_{o}\left(U^{r}\right) \leqq \operatorname{dim} g_{o}\left(\tilde{U}^{r}\right)$ 
pour tout sous-espace vectoriel ⿳亠㐅े $r$ dimensions $\tilde{U}^{r}$ de $V / W$.

Cette proposition résulte du Lemme 3.3 et du Lemme 3.4.

12 A partir de ce paragraphe, nous supposons que tous les objets géométriques soient analytiques réels. Tout ce que nous avons obtenu jusqu'à présent est encore valable en remplaçant le mot "différentiable" par "analytique réel". Nous nous proposons dans ce cas-là d'indiquer certains théorèmes qui ramènent le problème d'existence au problème algébrique.

Voici les théorèmes fondamentaux.

THÉORÈME 3.4. Soient $B$ une G-structure analytique et $\pi$-projetable et $R$ une équation différentielle analytique invariante par $B . \quad S$ 'il existe un relèvement $\sigma$ de $\tilde{\pi}_{0}: V_{0} \rightarrow V_{0} / W_{0}$ tel que

i) $\Phi_{\sigma}(B) \subset R$,

ii) la fonction structurale partielle $C_{\sigma}$ est nulle,

iii) $\mathrm{g}(\sigma) \subset \operatorname{Hom}\left(V_{0} / W_{0}, W_{0}\right)$ est involutif, g étant l'algèbre de Lie du groupe structural de $B$,

alors il existe, pour tout $y \in M$, une solution locale de $R$ passant par le point $y$.

Laissons la démonstration de ce théorème pour le moment.

THÉORÈme 3.5. Soit $\mathscr{L}$ un faisceau en algèbres de Lie analytique transitif et $\pi$-projetable et soit $R$ une équation différentielle analytique invariante par $\mathscr{L}$. Soit $y_{0} \in M$ et soit $\left(L, L^{0}, k(L)\right)$ le K-système d'algèbres de Lie attaché à $\mathscr{L}$ en $y_{0}$. S'il existe un relèvement $z_{0} \in R$ tel que

i) $\beta\left(z_{0}\right)=y_{0}$,

ii) la fonction structurale partielle $C_{z_{0}}$ de $\left(L, L^{0}, k(L)\right)$ est nulle,

iii) $\mathrm{g}\left(z_{0}\right)$ est involutif, g étant l'algèbre d'isotropie de $\left(L, L^{0}, k(L)\right)$, alors il existe, pour tout $y \in M$, une solution locale de $R$ passant par le point $y$.

En effet, nous pouvons passer la situation à celle de Théorème 3.4 en posant $\sigma=u_{0}^{-1} \circ z_{0} \circ t\left(u_{0}\right), u_{0}$ étant un élément de $P(\pi)$ vérifiant $\varpi\left(u_{0}\right)$ $=y_{0}$ (voir la démonstration du Théorème 3.3).

Pour démontrer le Théorème 3.4 , nous nous rappelons la théorie des systèmes différentiels extérieurs, (voir [3], [4], [8]).

Soit $P$ une variété, indiquons par $\Lambda(P)$ l'algèbre des formes différ- 
entielles extérieures de $P$ et par $d: \Lambda(P) \rightarrow \Lambda(P)$ l'opération différentielle extérieure. Un sous-ensemble $\Sigma$ de $\Lambda(P)$ est dit un système différentiel extérieur si et seulement si $\Sigma$ satisfait aux conditions suivantes:

1) $\Sigma$ est un idéal de $\Lambda(P), \quad$ 2) $d(\Sigma) \subset \Sigma$,

3) $\Sigma$ est homogène, i.e., $\Sigma=\Sigma^{(0)}+\Sigma^{(1)}+\cdots+\Sigma^{(m)}$

(la somme directe, $m=\operatorname{dim} P$ ), où $\Sigma^{(r)}=\Sigma \cap$ (l'espace vectoriel des formes de degré $r$ ),

4) $\Sigma$ est engendré par un nombre fini d'éléments de $\Sigma$. Remarquons que si $\Omega^{1}, \cdots, \Omega^{s}$ sont des formes de degré 1 sur $P$, elles déterminent le système différentiel extérieur engendré par $\Omega^{i}, d \Omega^{i}(i=1,2, \cdots, s)$. Nous ne considérerons qu'un tel système et par suite $\Sigma^{(0)}=\{0\}$.

Soit $G_{p}^{r}(P)$ la variété grassmannienne de tous les éléments plans à $r$ dimensions en $p \in P$, posons $G^{r}(P)=\cup_{p \in P} G_{p}^{r}(P) . G^{r}(P)$ est le fibré grassmannien sur $P$ avec la fibration canonique $\rho$. Un élément plan $E \in G^{r}(P)$ est dit intégral si et seulement si, pour tout $\theta^{(s)} \in \Sigma^{(s)}$ et tous $X_{1}, \cdots, X_{s} \in E, \theta^{(s)}\left(X_{1}, \cdots, X_{s}\right)=0$ est vérifié $(s=1,2, \cdots, m)$. Notons par $I^{r}(\Sigma)$ l'ensemble de tous les éléments plans intégrals de $\Sigma$. Pour tout $E \in G_{p}^{r}(P)(p \in P)$, désignons par $H(E)$ le sous-espace vectoriel de $T_{p}(P)$ de tous les éléments $X \in T_{p}(P)$ vérifiant $\theta\left(X, Y_{1}, \cdots, Y_{s-1}\right)=0$ pour tout $\theta \in \Sigma^{(s)}$, tous $Y_{1}, \cdots, Y_{s-1} \in E$ et tous $s=1,2, \cdots, m . \quad H(E)$ est appelé l'espace polair de $E$. Posons, pour tout $E \in G^{r}(P)$,

$$
t(E)=\operatorname{dim} P-\operatorname{dim} H(E)
$$

et, de plus,

$$
t_{s}(E)=\operatorname{Max}\left\{t\left(E^{\prime}\right) \mid E^{\prime} \in G^{s}(P) \text { avec } E^{\prime} \subset E\right\} \quad(s=0,1, \cdots, r) .
$$

Un élément plan intégral $E \in I^{r}(\Sigma)$ est dit ordinaire s'il existe un ouvert $\mathscr{N}$ de $G^{r}(P)$ tel que

i) $E \in \mathscr{N}$

ii) $I^{r}(\Sigma) \cap \mathscr{N}$ est un sous-fibré de $\mathscr{N}$ par rapport à la fibration $\left.\rho\right|_{\mathscr{N}}$

iii) $\operatorname{dim}\left(I^{r}(\Sigma) \cap \mathscr{N}\right)=\operatorname{dim} P+r(\operatorname{dim} P-r)-\sum_{s=0}^{r-1} t_{s}(E)$.

Considérons en outre une fibration $\alpha: P \rightarrow N$, où $N$ est une autre variété de dimension $n$. Un élément plan $E \in G_{p}^{n}(P)$ sera dit un $N$-élément plan si et seulement si l'application linéaire $\left.\alpha_{*}\right|_{E}: E \rightarrow T_{\alpha(p)}(N)$ est un isomorphisme de $E$ sur $T_{\alpha(p)}(N)$. Indiquons par $G(P, N)$ l'ensemble de tous les $N$-éléments plans; il est facile de démontrer que $G(P, N)$ 
s'identifie canoniquement avec la variété $J^{1}(P, \alpha)$ et que $G(P, N)$ est ouvert dans $G^{n}(P)$. Par conséquent, un $N$-élément plan intégral $E$ $\in I(\Sigma, N)=I^{n}(\Sigma) \cap G(P, N)$ est ordinaire si et seulement s'il existe un ouvert $\mathscr{N}^{\prime}$ de $G(P, N)$ tel que

i) $E \in \mathscr{N}^{\prime}$,

ii) $\quad I(\Sigma, N) \cap \mathscr{N}^{\prime}$ est un sous-fibré de $\mathscr{N}^{\prime}$ par rapport à la fibration $\left.\rho\right|_{\mathcal{N}^{\prime}}$,

iii) $\operatorname{dim}\left(I(\Sigma, N) \cap \mathscr{N}^{\prime}\right)=\operatorname{dim} P+n(\operatorname{dim} P-n)-\sum_{s=0}^{n-1} t_{s}(E)$. Nous disons que le système $(\Sigma, P, \alpha, N)$ est en involution à un point $p \in P$ si et seulement si les conditions suivantes sont vérifiées:

1) il existe un $N$-élément plan intégral $E$ avec $\rho(E)=p$,

2) tout $N$-élément plan intégral en $p$ est ordinaire.

Une section locale $s$ de $\alpha$ est dit une solution locale de $\Sigma$ si $s^{*} \theta=0$ est vérifié pour tout $\theta \in \Sigma$. En vertu du Théorème de Cartan-Kähler, nous pouvons retrouver la proposition suivante, (voir [3], [4]).

Proposition 3.3. Supposons que $(\Sigma, P, \alpha, N)$ est en involution à un point $p \in P$. Alors il existe une solution locale de $\Sigma$ passant par le point $p$.

A l'aide de cette proposition, nous allons démontrer le Théorème 3.4. Considérons le système différentiel extérieur engendré par $\Omega^{\sigma}$ et $d \Omega^{\sigma}, \Sigma\left(\Omega^{o}\right)$. On peut constater d'abord que tous les $N$-éléments plans de $(B, \pi \circ \varpi, N)$ sont représentés par les éléments de $J^{1}(B, \pi \circ \varpi)$ :

$$
E(Y)=Y\left(T_{x}(N)\right) \quad\left(Y \in J^{1}(B, \pi \circ \varpi), x=\pi \circ \varpi \circ \beta(Y)\right) .
$$

Lemme 3.5. Pour tous $Y \in R^{o}(B), A \in \mathfrak{g}$ et tous $\xi, \eta \in V_{0} / W_{0}$,

$$
\begin{aligned}
& \left(d \Omega^{o}\right)_{u}\left(\left(Y(t(u)(\xi))+A_{u}^{*}\right) \wedge Y(t(u)(\eta))\right) \\
& \quad=\bar{C}_{o}(Y)(\xi \wedge \eta)-\phi_{\sigma}(A)(\eta)(u=\beta(Y)) .
\end{aligned}
$$

Ce lemme résulte immédiatement de la définition de $\bar{C}_{\sigma}(2.13)$ et du Lemme 2.5.

Lemme 3.6. Pour que $Y \in J^{1}(B, \pi \circ \varpi)$ s'identifie avec un $N$-élément plan intégral de $\Sigma\left(\Omega^{\sigma}\right)$, il faut et il suffit que $Y \in R^{\sigma}(B)$ et $\bar{C}_{\sigma}(Y)=0$.

En effet, supposons que $Y$ donne un $N$-élément plan intégral de $\Sigma\left(\Omega^{\sigma}\right)$, c'est-à-dire que

i) $\Omega_{u}^{o}(Y(v))=0$, 
ii) $\left(d \Omega^{\circ}\right)_{u}\left(Y(v) \wedge Y\left(v^{\prime}\right)\right)=0 \quad(u=\beta(Y))$

pour tous $v, v^{\prime} \in T_{x}(N)(x=\pi \circ \varpi(u))$. D'après le Lemme 3.2, i) signifie qu'il existe $Y^{\prime} \in R^{\sigma}(B)$ et $A \in \mathfrak{g}$ tels que $Y(v)=Y^{\prime}(v)+A_{u}^{*}$. On a par définition

$$
\begin{aligned}
0=\theta_{u}\left(A_{u}^{*}\right) & =\theta_{u}\left(Y(v)-Y^{\prime}(v)\right) \\
& =\theta_{u}(Y(v))-\sigma\left(t(u)^{-1}(v)\right)
\end{aligned}
$$

et par suite, $\theta_{u}(Y(v))=\sigma\left(t(u)^{-1}(v)\right)$. Ceci démontre que $Y \in R^{o}(B)$. Ensuite, ii) signifie que $\bar{C}_{\sigma}(Y)=0$. La réciproque sera évidente.

LEMME 3.7. Soit $U^{r}$ un sous-espace vectoriel à $r$ dimensions de $V_{0} / W_{0}$. Si $U^{r}$ est générique par rapport à $\mathfrak{g}(\sigma)$,

$$
t_{r}(E(Y))=\operatorname{dim} B-\operatorname{dim} N-\operatorname{dim} \mathrm{g}_{o}\left(U^{r}\right)
$$

pour tout $Y \in R^{\sigma}(B)$ vérifiant $\bar{C}_{\sigma}(Y)=0$.

En effet, indiquons par $H\left(Y, U^{r}\right)$ l'espace polaire de $E^{r}=Y\left(U^{r}\right)$, i.e., $H\left(Y, U^{r}\right)=\left\{Z \in T_{u}(B) \mid \Omega_{u}^{\sigma}(Z)=0,\left(d \Omega^{o}\right)_{u}\left(Z \wedge Z^{\prime}\right)=0\right.$ pour tout $\left.Z^{\prime} \in E^{r}\right\}$.

D'apès le Lemme 3.2, $Z$ doit être de la forme $Z=Y(v)+A_{u}^{*}(A \in \mathfrak{g}$, $\left.v=\pi_{*} \widetilde{\varpi}_{*}(Z)\right)$. Puis d'après le Lemme 3.5 , on a

$$
\left(d \Omega^{\sigma}\right)_{u}\left(Z \wedge Z^{\prime}\right)=-\phi_{\sigma}(A)(\eta)=0
$$

en posant $Z^{\prime}=Y(t(u)(\eta))\left(\eta \in V_{0} / W_{0}\right)$. Par suite, $H\left(Y, U^{r}\right)$ est isomorphe à la somme directe $\left(V_{0} / W_{0}\right) \oplus \mathrm{g}_{\sigma}\left(U^{r}\right)$, on a donc

$$
t\left(Y\left(U^{r}\right)\right)=\operatorname{dim} B-\operatorname{dim} N-\operatorname{dim} \mathrm{g}_{\sigma}\left(U^{r}\right) .
$$

A l'aide du Lemme 3.4, on peut démontrer aisément que $t\left(Y\left(U^{r}\right)\right)$ $\geqq t\left(Y\left(\widetilde{U^{r}}\right)\right)$ pour tout sous-espace vectoriel à $r$ dimensions $\widetilde{U^{r}}$ de $V_{0} / W_{0}$. Donc le lemme est établi.

Maintenant fixons un élément $y_{0} \in M$ et un élément $u_{0} \in B$ avec $\widetilde{\sigma}\left(u_{0}\right)=y_{0}$. D'après la Proposition 2.13, il existe un élément $Y_{0} \in R^{\sigma}(B)$ tel que $\beta\left(Y_{0}\right)=u_{0}$ et $\bar{C}_{\sigma}\left(Y_{0}\right)=0$. Par suite, il existe un $N$-élément plan intégral de $\Sigma\left(\Omega^{\sigma}\right)$ en $u_{0}$ (cf. Lemme 3.6). Donc il suffit de démontrer que tout $Y \in R^{o}(B)$ vérifiant $\beta(Y)=u_{0}$ et $\bar{C}_{\sigma}(Y)=0$ est ordinaire.

Puisque $C_{\sigma}$ est nulle sur $B$, il existe, d'après la Proposition 2.14, une section locale $\tau$ de $\beta: R^{\sigma}(B) \rightarrow B$ définie autour du point $u_{0}$ telle que $\bar{C}_{\sigma}(\tau(u))=0$ pour tout $u \in U(\tau)$. Nous pouvons supposer que $\tau$ vérifie 
aussi $\tau\left(u_{0}\right)=Y$.

Considérons l'application

$$
\Psi: U(\tau) \times I^{o}(\mathrm{~g}) \rightarrow I\left(\Sigma\left(\Omega^{o}\right), N\right) \cap \beta^{-1}(U(\tau))
$$

définie par $\Psi(u, A)=A * \tau(u)\left((u, A) \in U(\tau) \times I^{\sigma}(\mathrm{g})\right)$. On a $\beta \circ \Psi(u, A)=u$ et $\Psi\left(u_{0}, 0\right)=Y$. Il est facile de démontrer, d'après la Proposition 2.11, que $\Psi$ est injective. Soit $Y^{\prime}$ un élément de $I\left(\Sigma\left(\Omega^{\sigma}\right), N\right) \cap \beta^{-1}(U(\tau))$. D'après la même Proposition 2.11, il existe $A \in \operatorname{Hom}\left(V_{0} / W_{0}, \mathfrak{g}\right)$ tel que $Y^{\prime}$ $=A * \tau(u)\left(u=\beta\left(Y^{\prime}\right)\right)$. On a

$$
\begin{aligned}
\delta_{\sigma} A & =-\bar{C}_{o}(\tau(u))+\delta_{\sigma} A \\
& =-\bar{C}_{o}\left(Y^{\prime}\right)=0
\end{aligned}
$$

et par suite, $A \in I^{\sigma}(\mathrm{g})$. Ceci démontre que $\Psi$ est surjective. Comme $R^{\sigma}(B)$ est localement représenté par le produit de $U(\tau)$ et $\operatorname{Hom}\left(V_{0} / W_{0}, \mathrm{~g}\right)$, $\mathscr{M}=I\left(\Sigma\left(\Omega^{\sigma}\right), N\right) \cap \beta^{-1}(U(\tau))$ est envisagé par l'application bijective $\Psi$ comme un sous-fibré de $R^{\sigma}(B) \cap \beta^{-1}(U(\tau))$ par rapport à la fibration $\beta$. Il en résulte que $\mathscr{M}$ est un sous-fibré de $\beta^{-1}(U(\tau))$.

Comme $g(\sigma)$ est involutif, il existe une série de sous-espaces vectoriels génériques $\{0\}=U^{0} \subset U^{1} \subset \cdots \subset U^{n}=V_{0} / W_{0}$ telle que $\operatorname{dim} I^{\sigma}(\mathfrak{g})$ $=\sum_{r=0}^{n-1} \operatorname{dim} \mathfrak{g}_{o}\left(U^{r}\right)$ et $t_{r}(E(Y))=\operatorname{dim} B-\operatorname{dim} N-\operatorname{dim} \mathfrak{g}_{o}\left(U^{r}\right) \quad(r=0,1$, $\cdots, n-1$ ) (cf. Proposition 3.2 et Lemme 3.7). Calculons $\operatorname{dim} \mathscr{M}$,

$$
\begin{aligned}
\operatorname{dim} \mathscr{M} & =\operatorname{dim} B+\operatorname{dim} I^{o}(\mathfrak{g}) \\
& =\operatorname{dim} B+\sum_{r=0}^{n-1} \operatorname{dim} \mathfrak{g}_{o}\left(U^{r}\right) \\
& =\operatorname{dim} B+\sum_{r=0}^{n-1}\left(\operatorname{dim} B-\operatorname{dim} N-t_{r}(E(Y))\right. \\
& =\operatorname{dim} B+n(\operatorname{dim} B-n)-\sum_{r=0}^{n-1} t_{r}(E(Y)) .
\end{aligned}
$$

Ceci démontre que $E(Y)$ est ordinaire. Le Théorème 3.4 résute donc de la Proposition 3.3 et de la Proposition 3.1. c.q.f.d.

13 Maintenant nous allons appliquer la théorie générale à la géométrie des $G$-structures.

Soient $N_{1}, N_{2}$ des variétés analytiques réelles. Supposons que $\operatorname{dim} N_{1}$ $=\operatorname{dim} N_{2}=n . \quad$ Soit $G$ un sous-groupe de Lie de $G L(n, R)$ et soient $B_{1}$, $B_{2}$ des $G$-structures sur $N_{1}$ et $N_{2}$ respectivement. $B_{1}$ et $B_{2}$ déterminent l'équation différentielle du premier ordre $R\left(B_{1}, B_{2}\right)$ sur $\left(N_{1} \times N_{2}, \pi_{1}, N_{1}\right)$, $\pi_{1}$ étant la projection canonique (cf. $\S 2$, Exemple II). $R\left(B_{1}, B_{2}\right)$ est une équation invariante par le pseudo-groupe des automorphismes locaux de 
$B_{1}$ et de $B_{2}$. Remarquons que $B_{1} \times B_{2}$ est plongé canoniquement dans le fibré des repères de $N_{1} \times N_{2}$. Définissons l'application linéaire $\sigma: \boldsymbol{R}^{n}$ $\rightarrow \boldsymbol{R}^{2 n}=\boldsymbol{R}^{n} \oplus \boldsymbol{R}^{n}$ par $\sigma(\xi)=(\xi, \xi)\left(\xi \in \boldsymbol{R}^{n}\right) ; \sigma$ est un relèvement par rapport à la projection canonique $\pi_{0}: \boldsymbol{R}^{2 n} \rightarrow \boldsymbol{R}^{n}$.

LEMME 3.8. $\Phi_{\sigma}\left(B_{1} \times B_{2}\right)=R\left(B_{1}, B_{2}\right)$.

En effet, on a d'abord $t(u, v)=u$ pour tous $u \in B_{1}, v \in B_{2}$ (cf. 2.5) et $\Phi_{o}(u, v)(X)=\left(X, v \circ u^{-1}(X)\right)$ pour tout $X \in T_{x}\left(N_{1}\right)\left(x=\varpi_{1}(u)\right)$. Par suite, $\Phi_{o}(u, v)$ s'identifie avec $v \circ u^{-1}$. Il en résulte que $\Phi_{o}(u, v)\left(\left(B_{1}\right)_{x}\right)=\left(B_{2}\right)_{y}$ $\left(y=\varpi_{2}(v)\right)$. Soit $z \in R\left(B_{1}, B_{2}\right)$ avec $\alpha(z)=x, \beta(z)=y$. Prenons $u \in B_{1}$, $v \in B_{2}$ avec $\widetilde{\varpi}_{1}(u)=x, \varpi_{2}(v)=y$ et posons $a=v^{-1} \circ z \circ u$. Comme $z \circ u$ appartient à $B_{2}, v \circ a$ est un élément de $B_{2}$. Donc on obtient $\Phi_{o}(u, v \circ a)=z$.

Soient $\theta_{1}, \theta_{2}$ les formes canoniques sur $B_{1}$ et $B_{2}$ respectivement et $\theta$ la forme canonique sur $B_{1} \times B_{2}$. Il est immédiat de constater que $\theta$ coïncide avec $\theta_{1}-\theta_{2}$ et que $\Omega^{o}=\theta_{2}-\theta_{1}$. Soit $R^{o}\left(B_{1} \times B_{2}\right)$ l'équation différentielle sur $\left(B_{1} \times B_{2}, \varpi_{1}, N_{1}\right)$ déterminée par $\Omega^{\circ}$ (cf. $\S 2$, Exemple III et Proposition 2.10). Définissons l'application $F: J^{1}\left(B_{1}, \varpi_{1}\right) \times J^{1}\left(B_{2}, \varpi_{2}\right)$ $\rightarrow R^{\circ}\left(B_{1} \times B_{2}\right)$ par la formule:

$$
F\left(X_{1}, X_{2}\right)(Y)=\left(X_{1}(Y), X_{2} \circ u_{2} \circ u_{1}^{-1}(Y)\right) \quad\left(u_{i}=\beta\left(X_{i}\right), i=1,2\right)
$$

pour tous $X_{i} \in J^{1}\left(B_{i}, \varpi_{i}\right), i=1,2$, et tout $Y \in T_{x}\left(N_{1}\right)\left(x=\widetilde{\varpi}_{1}\left(u_{1}\right)\right)$. On peut constater que $F$ possède l'image dans $R^{o}\left(B_{1}, B_{2}\right)$.

LEMME 3.9. F est surjective.

En effet, soit $Y \in R^{o}\left(B_{1} \times B_{2}\right)$ avec $\beta(Y)=\left(u_{1}, u_{2}\right)$. Comme pour tout $X \in T_{x}\left(N_{1}\right)\left(x=\varpi_{1}\left(u_{1}\right)\right), Y(X)$ est un élément de $T_{\left(u_{1}, u_{2}\right)}\left(B_{1} \times B_{2}\right)=T_{u_{1}}\left(B_{1}\right)$ $\oplus T_{u_{2}}\left(B_{2}\right)$, nous pouvons partager $Y(X)$ en deux: $Y(X)=\left(Y_{1}(X), Y_{2}(X)\right)$ $\left(Y_{i}(X) \in T_{u_{i}}\left(B_{i}\right), i=1,2\right)$. Il est clair que $Y_{i}, i=1,2$, sont des application linéaires. Posons $X_{1}=Y_{1}$ et $X_{2}=Y_{2} \circ u_{1} \circ u_{2}^{-1}$. Alors on obtient $X_{i} \in J^{1}\left(B_{i}, \varpi_{i}\right), i=1,2$, et par suite, $F\left(X_{1}, X_{2}\right)=Y$.

LEMME 3.10. La fonction structurale partielle $C_{\text {o }}$ sur $B_{1} \times B_{2}$ coïncide avec $C_{2}-C_{1}, C_{i}$ étant la fonction structurale de $B_{i}(i=1,2)$.

En effet, pour $X_{i} \in J^{1}\left(B_{i}, \varpi_{i}\right), i=1,2, \xi, \eta \in R^{n}$, on a

$$
\begin{aligned}
\bar{C}_{o}\left(F\left(X_{1}, X_{2}\right)\right)(\xi \wedge \eta) & =\left(d \Omega^{\circ}\right)\left(\left(X_{1} \circ u_{1}(\xi), X_{2} \circ u_{2}(\xi)\right) \wedge\left(X_{1} \circ u_{1}(\eta), X_{2} \circ u_{2}(\eta)\right)\right. \\
& =\left(d \theta_{2}\right)_{u_{2}}\left(X_{2} \circ u_{2}(\xi) \wedge X_{2} \circ u_{2}(\eta)\right)
\end{aligned}
$$




$$
\begin{aligned}
& -\left(d \theta_{1}\right)_{u_{1}}\left(X_{1} \circ u_{1}(\xi) \wedge X_{1} \circ u_{1}(\eta)\right) \\
= & \bar{C}_{2}\left(X_{2}\right)(\xi \wedge \eta)-\bar{C}_{1}\left(X_{1}\right)(\xi \wedge \eta)
\end{aligned}
$$

et par suite, $C_{\sigma}\left(u_{1}, u_{2}\right)=C_{2}\left(u_{2}\right)-C_{1}\left(u_{1}\right)$.

Soit $g$ l'algèbre de Lie de $G$. Désignons par $\tilde{g}$ l'algèbre de Lie $d u$ groupe structural de $B_{1} \times B_{2}$, i.e., $\tilde{\mathrm{g}}=\mathrm{g} \oplus \mathrm{g}$ (la somme directe).

LEMME 3.11. $\tilde{\mathrm{g}}(\sigma)=\mathfrak{g}$.

En effet, soit $A=\left(A_{1}, A_{2}\right) \in \tilde{g}\left(A_{i} \in \mathfrak{g}, i=1,2\right)$, on a

$$
\begin{aligned}
\phi_{\sigma}(A)(\xi) & =A \circ \sigma(\xi)-\sigma \circ \pi_{0} \circ A \circ \sigma(\xi) \\
& =\left(A_{1}(\xi), A_{2}(\xi)\right)-\sigma\left(A_{1}(\xi)\right) \\
& =\left(0,\left(A_{2}-A_{1}\right)(\xi)\right)
\end{aligned}
$$

pour tout $\xi \in \boldsymbol{R}^{n}$.

Nous pouvons démontrer le théorème suivant qui a été trouvé par Singer et Sternberg (voir [11], Chap, III).

THÉORÈmE 3.6. Soient $B_{1}, B_{2}$ des G-structures sur $N_{1}$ et $N_{2}$ respectivement et $C_{1}, C_{2}$ les fonctions structurales de $B_{1}$ et $B_{2}$ respectivement. Si les conditions suivantes sont vérifiées:

1) $C_{1}$ et $C_{2}$ sont constantes et $C_{1}=C_{2}$,

2) l'algèbre de Lie de $G, \mathfrak{g}$, est involutive, alors $B_{1}$ et $B_{2}$ sont localement isomorphes.

En effet, d'après le Lemme $3.10,1$ ) signifie que la fonction $C_{\sigma}$ est nulle sur $B_{1} \times B_{2}$; d'après le Lemme $\left.3.11,2\right)$ signifie que $\tilde{g}(\sigma)$ est involutive. Ce théorème résulte donc du Théorème 3.4.

Soit $N$ une variété de dimension $n$ et soit $B$ une $G$-structure sur $N$. Supposons que la fonction structurale $C$ de $B$ soit nulle. Désignons par $R(B)$ l'ensemble de tous les éléments $z$ de $J^{1}(B, \varpi)$ vérifiant la relation $(d \theta)_{u}(z(X), z(Y))=0(u=\beta(z))$ pour tous $X, Y \in T_{x}(N)(x=\varpi(u)), \theta$ étant la forme canonique sur $B$. Il se trouve que $R(B)$ est une équation différentielle du premier ordre $\operatorname{sur}(B, \varpi, N)$ invariante par le pseudogroupe des automorphismes locaux de $B$. Pour qu'il existe, pour tout point $x \in N$, une solution locale de $R(B)$ définie autour du point $x$, il faut et il suffit que $B$ est plate (cf. Definition 3.1).

Pour tout $z \in R(B)$, nous pouvons définir l'élément $H(z)$ de $F(B)$, le fibré des repères de $B$, par les formules: 


$$
H(z)(\xi)=z \circ u(\xi), \quad H(z)(A)=A_{u}^{*} \quad(u=\beta(z))
$$

pour tout $\xi \in \boldsymbol{R}^{n}$ et tout $A \in \mathfrak{g}$, l'algèbre de Lie de $G$. Soit $\mathfrak{g}^{(1)}$ le prolongement algébrique d'ordre 1 de g. Posons

$$
G^{(1)}=\left\{\tilde{X} \in G L\left(\boldsymbol{R}^{n} \oplus \mathrm{g}\right) \tilde{X}(\xi, A)=(\xi, X(\xi)+A), X \in \mathfrak{g}^{(1)}\right\}
$$

et $B^{(1)} \equiv H(R(B))$. Alors $B^{(1)}$ est une $G^{(1)}$-structure sur $B$, le prolongement géométrique d'ordre 1 de $B$ (cf. [10]). Définissons l'application $\sigma: \boldsymbol{R}^{n} \rightarrow \boldsymbol{R}^{n} \oplus \mathrm{g}$ par $\sigma(\xi)=(\xi, 0)\left(\xi \in \boldsymbol{R}^{n}\right)$. Il est facile de démontrer que $\Phi_{\sigma}\left(B^{(1)}\right)=R(B)$ et que $\phi_{\sigma}(\tilde{g})=\mathfrak{g}^{(1)}$, $\tilde{g}$ étant l'algèbre de Lie de $G^{(1)}$. Le Théorème 3.4 s'applique à cette situation. Lorsque $B$ est une structure riemannienne, i.e., $\mathfrak{g}=\mathfrak{o}(n)$, la fonction structurale partielle $C_{\sigma}$ sur $B^{(1)}$ est envisagée comme la courbure de cette structure riemannienne et $\mathfrak{g}^{(1)}=0$. Dans ce cas-là, le Théorème 3.2 montre un des théorèmes fondamentaux de la géométrie riemannienne: Si la courbure de l'espace de Riemann $N$ est nulle, $N$ est un espace localement euclidien.

\section{BIBLIOGRAPHIE}

[1] E. Cartan, Leçons sur les invariants intégraux, Hermann, Paris, 1922.

[2] - Sur la possibilité de plonger un espace riemannien donné dans un espace euclidien, Ann. Soc. Polon. Math. 6 (1927), 1-7.

[ 3 ] — Les systèmes différentiels extérieurs et leurs applications géométriques, Hermann, Paris, 1945.

[ 4 ] J. Dieudonné, Eléments d'analyse, Tome IV, Gauthier-Villars, Paris, 1971.

[ 5 ] H. Goldschmidt, Existence theorems for analytic linear partial differential equations, Ann. of Math. 86 (1967), 246-270.

[6] - Integrability criteria for systems of nonlinear partial differential equations, J. Differential Geometry 1 (1969), 269-307.

[7] S. Kobayashi and T. Nagano, On filtered Lie algebras and geometric structures V, J. Math. Mech. 15 (1966), 315-328.

[8] M. Kuranishi, Lectures on exterior differential systems, Tata Institute of Fundamental Research, Bombay, 1962.

[ 9 ] S. Lie, Zur allgemeinen Theorie der partiellen Differentialgleichungen beliebiger Ordnung, Leipzig Ber. 47 (1895), 53-128; Gesam. Abh. Bd. VI, 320-384.

[10] S. Sternberg, Lectures on differential geometry, Prentice-Hall, Englewood Cliffs, N.J., 1964.

[11] I. M. Singer and S. Sternberg, The infinite group of Lie and Cartan, J. Analyse Math. 15 (1965), 1-144.

Institut de Mathématiques, Université de Nagoya 